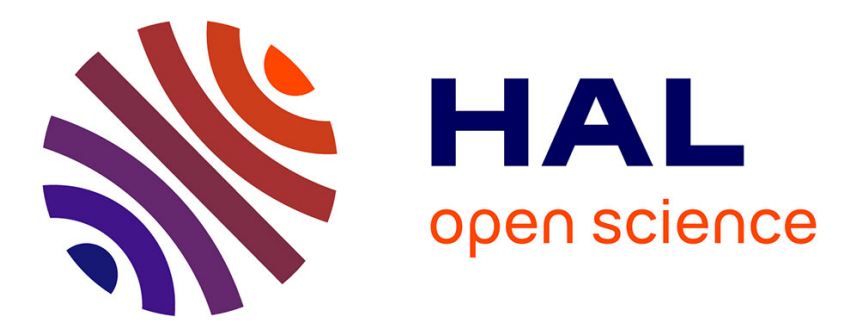

\title{
A useful magnesium reagent for the preparation of 1,1-difluoro-2-hydroxyphosphonates from diethyl bromodifluoromethylphosphonate via a metal-halogen exchange reaction
}

\author{
Rachel Waschbüsch, Mohammad Samadi, Philippe Savignac
}

\section{To cite this version:}

Rachel Waschbüsch, Mohammad Samadi, Philippe Savignac. A useful magnesium reagent for the preparation of 1,1-difluoro-2-hydroxyphosphonates from diethyl bromodifluoromethylphosphonate via a metal-halogen exchange reaction. Journal of Organometallic Chemistry, 1997, 529 (1-2), pp.267-278. 10.1016/S0022-328X(96)06541-2 . hal-03166507

\section{HAL Id: hal-03166507 https://hal.science/hal-03166507}

Submitted on 11 Mar 2021

HAL is a multi-disciplinary open access archive for the deposit and dissemination of scientific research documents, whether they are published or not. The documents may come from teaching and research institutions in France or abroad, or from public or private research centers.
L'archive ouverte pluridisciplinaire $\mathbf{H A L}$, est destinée au dépôt et à la diffusion de documents scientifiques de niveau recherche, publiés ou non, émanant des établissements d'enseignement et de recherche français ou étrangers, des laboratoires publics ou privés. 


\title{
A useful magnesium reagent for the preparation of 1,1-difluoro-2- hydroxyphosphonates from diethyl bromodifluoromethylphos- phonate via a metal-halogen exchange reaction
}

\author{
Rachel Waschbüsch, Mohammad Samadi and Philippe Savignac \\ Hétéroéléments et Coordination, URA CNRS 1499, DCPH, Ecole Polytechnique, 91128 Palaiseau Cedex \\ (France)
}

\begin{abstract}
When $(\mathrm{EtO})_{2} \mathrm{P}(\mathrm{O}) \mathrm{CF}_{2} \mathrm{Br}(\mathbf{1})$ is treated with isopropylmagnesium chloride in $\mathrm{THF}$ at low temperature it gives a magnesium species (2) which undergoes reactions with strong electrophiles ( $\mathrm{HCl}$, $\mathrm{TMSCl}$, halogens, aldehydes and ketones). The formation of products strongly depends on the reaction conditions. With 1.5 equivalents of $(2)$ between $-78^{\circ} \mathrm{C}$ and $0^{\circ} \mathrm{C}$, a conversion of more than $90 \%$ of aldehydes and ketones into 2-hydroxyphosphonates (7) and (8) can be achieved. These compounds (7) and (8) in the presence of base (NaH, LDA) are rearranged into 2,2-difluoroethylphosphates without concomitant formation of 1,1-difluoroolefines.
\end{abstract}

Key words : Isopropylmagnesium chloride, Metal-halogen exchange, Bromodifluorophosphonate, 1,1Difluoro-2-hydroxyphosphonate.

\section{Introduction}

The metal-halogen exchange reaction is a valuable tool in organic synthesis, since it can be performed under mild conditions and usually with great efficiency [1]. In the chemistry of phosphonates we started to make use of the metal-halogen reaction with diethyl trichloromethylphosphonate which has been tested as a representative compound [2]. The successive exchange reactions of the three chlorine atoms by treatment with butyllithium and an appropriate electrophile in THF was used for the synthesis of functionalized phosphonates without any complications. With the use of isopropylmagnesium chloride the same trichloromethyl phosphonate can undergo the exchange reaction of only one chlorine atom to give the desired diethyl dichloromethylphosphonate [3]. To our knowledge it is still the best method for obtaining pure dichloromethylphosphonate in high yield. As we were developing the metal-halogen reaction as a synthetic tool, we became interested in the extension of the reductive dehalogenation reaction to diethyl bromodifluoromethylphosphonate $\mathbf{1}$ as well as in exploiting the synthetic potential of the magnesium species. Such a metal-bromine exchange reaction had already been performed on this reagent with $\mathrm{Li}, \mathrm{Zn}$ and $\mathrm{Cd}$ as metal [4], we chose to perform it with $\mathrm{Mg}$ under mild conditions. 


\section{Results}

Our study anticipated an easy access to diethyl bromodifluoromethylphosphonate 1. This reagent was first prepared by Burton and Flynn via a Michaelis-Arbuzov type reaction from dibromodifluoromethane $\left(\mathrm{CF}_{2} \mathrm{Br}_{2}\right)$ and triethylphosphite $\left((\mathrm{EtO})_{3} \mathrm{P}\right)$ in refluxing $\mathrm{Et}_{2} \mathrm{O}$ [5]; a long reaction time was required $(24 \mathrm{~h})$. Later the same reaction was reported as "a violent reaction which blew out the addition funnel, nitrogen inlet and stopper. This explosion could be controlled by running the reaction in a steel bomb" [6].

For our part we found that the synthesis of $\mathbf{1}$ could be achieved on a molar scale and in safe conditions by slow addition of pure $(\mathrm{EtO})_{3} \mathrm{P}$ to a solution of $\mathrm{CF}_{2} \mathrm{Br}_{2}$ in refluxing THF. Under these conditions $\mathrm{CF}_{2} \mathrm{Br}_{2}$ reacts readily, cleanly and quantitatively with (EtO) ${ }_{3} \mathrm{P}$. A ${ }^{31} \mathrm{P}$ NMR spectroscopic investigation of the reaction mixture revealed that half an hour after the end of the addition $(\mathrm{EtO})_{3} \mathrm{P}$ was no longer detected and the only phosphorus species observed in the reaction mixture was diethyl bromodifluoromethylphosphonate 1 ( $\left.\delta{ }^{31} \mathrm{P}(\mathrm{THF})-0.4\right)$. A number of 1,1,1-trihalogenated phosphonates have been conveniently prepared according to this process which can be connected unambiguously to the Michaelis-Arbuzov reaction [7]. However among all the mechanistic pathways which are believed to be involved (ionic, radical and monoelectronic transfer) only the halophylic substitution $\left(\mathrm{SNCl}^{+}\right)$has been retained as the major contribution [8].

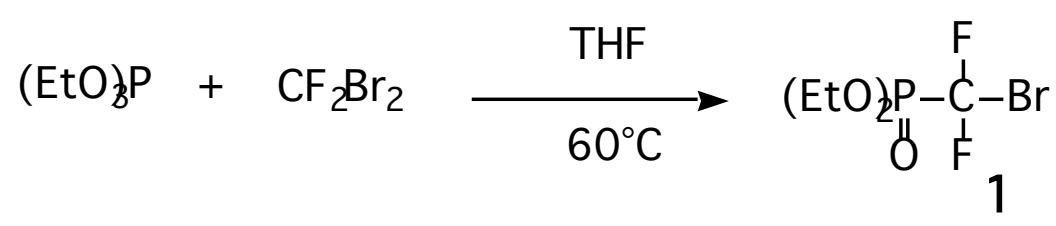

Satisfied with the preparation of $\mathbf{1}$, which was repeated with equal success several times, we decided to examine its reactivity. Diethyl bromodifluoromethylphosphonate $\mathbf{1}$ was treated at low temperature in $\mathrm{THF}$ with 1.1 equiv. of iPrMgCl. The magnesium-bromine exchange reaction was complete and the resultant magnesium compound $\mathbf{2}$ was obtained as a colorless, clear solution which could be stored at low temperature without any change. It is well known that diethyl lithiodifluoromethylphosphonate has to be kept at low temperature to prevent the thermal dissociation of the anion [4n, 9] when by contrast the organocadmium and organozinc reagents are remarkably stable at room temperature [4a, 4h]. The organomagnesium has an intermediate thermal stability. Effectively, when a cold solution of $\mathbf{2}$ in THF was slowly heated, the solution remained colorless until $-40^{\circ} \mathrm{C}$ and then turned progressively brown. This transformation was monitored by following the change in the ${ }^{31} \mathrm{P}$ NMR spectra of samples taken at various temperatures from $-40^{\circ} \mathrm{C}$ and hydrolysed in acidic medium. They revealed a 
decreasing intensity of the triplet corresponding to $\mathbf{3}$ indicating a slow decomposition of the magnesium species 2 .

The reactions of $\mathbf{2}$ were necessarily conducted at low temperature in order to prevent the anticipated decomposition. 2 reacts only with strong electrophiles such as aldehydes, ketones, halogens, chlorosilanes and mineral acid. This restricted reactivity is due to the poor nucleophilicity of the highly stabilized anion which moreover is thermally unstable above $40^{\circ} \mathrm{C}$. Therefore, it seemed necessary to find out how the reaction conditions influenced yields and reactivities. Firstly we decided to examine two good electrophiles, the proton and chlorotrimethylsilane.

The production of diethyl difluoromethylphosphonate $\mathbf{3}$ strongly depended on the proton source. $\mathrm{H}_{2} \mathrm{O}$ protonated the magnesium species 2 with only a reasonable yield (75\%). It was clear that the strongly electron-withdrawing difluoro group considerably activated the phosphoryl group which is very sensitive to nucleophilic attacks [9]. A fast reaction with the electrophile at low temperature was required and thus the use of a dilute hydrochloric acid solution was preferred.

The complete conversion of $\mathbf{2}$ into $\mathbf{3}$ on preparative scale was achieved by pouring at low temperature a solution of $\mathbf{2}$ in a cold, stirred biphasic mixture of $3 \mathrm{M}$ hydrochloric acid and $\mathrm{CH}_{2} \mathrm{Cl}_{2}$; with these conditions 3 could be isolated in a pure form and in good yield (85\%). With chlorotrimethylsilane, the magnesium reagent $\mathbf{2}$ also underwent complete conversion into silylated compound to give $\mathbf{4}$ in excellent yield (90\%) after treatment in acidic medium.

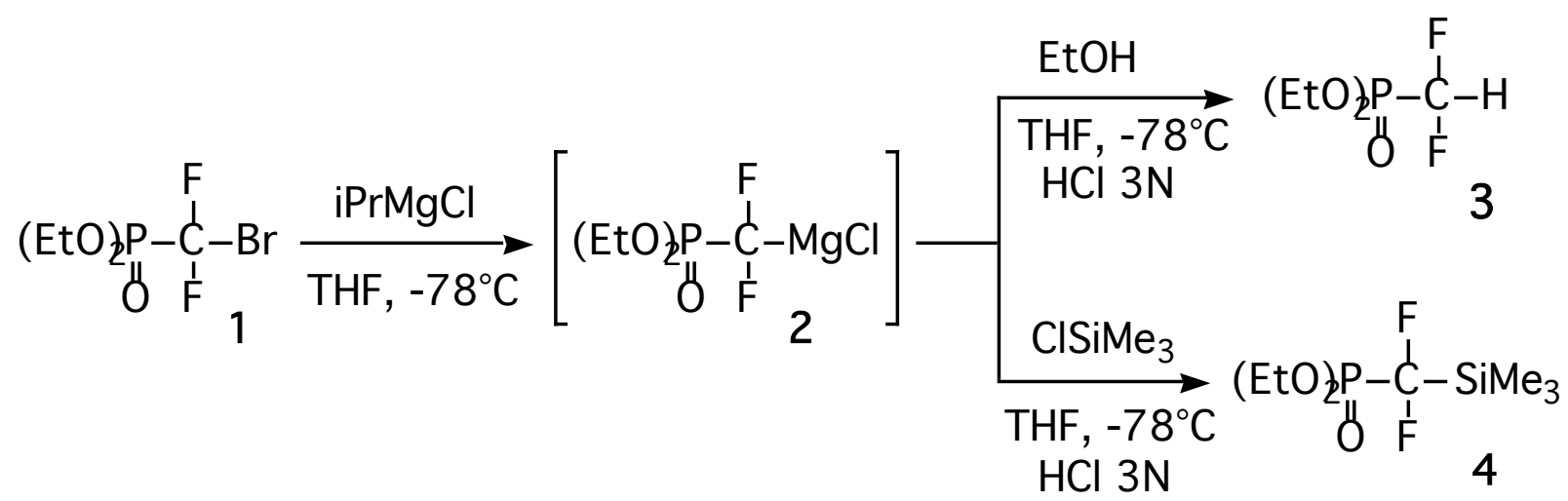

To get further insight in the synthetic potential of the magnesium species 2 , we also examined the reactivity of 2 with halogens $\left(\mathrm{I}_{2}\right)$ and halogenating agents $\left(\mathrm{C}_{2} \mathrm{Cl}_{6}\right)$. With iodine in THF at low temperature iodination remained the main reaction, ( $\left.\delta{ }^{31} \mathrm{P}(\mathrm{THF})-1.4\right)(80 \%)$, but two by-products could be detected, we assumed that these compounds were $\mathbf{3}\left(\delta{ }^{31} \mathrm{P} 4.9\right)$ and the difluoromethyl bis(diethylphosphonate) $\left(\delta{ }^{31} \mathrm{P}(\mathrm{THF}) 3.4\right)$. After acidic work-up at low temperature the iododifluoromethylphosphonate (6) was isolated and purified by distillation (48\%). With hexachloroethane at low temperature formation of chlorodifluoromethyl-phosphonate was the main reaction and the synthesis of (5) could be 
achieved with reasonable yield (60\%). This method of preparation of $\mathbf{5}$ and $\mathbf{6}$ is the most practical because attempts to prepare the compounds by reaction of $\mathrm{Cl}^{-}$or $\mathrm{I}^{-}$with $\mathbf{1}$ would give only dealkylation products by attack of the nucleophile on the phosphonate esters.

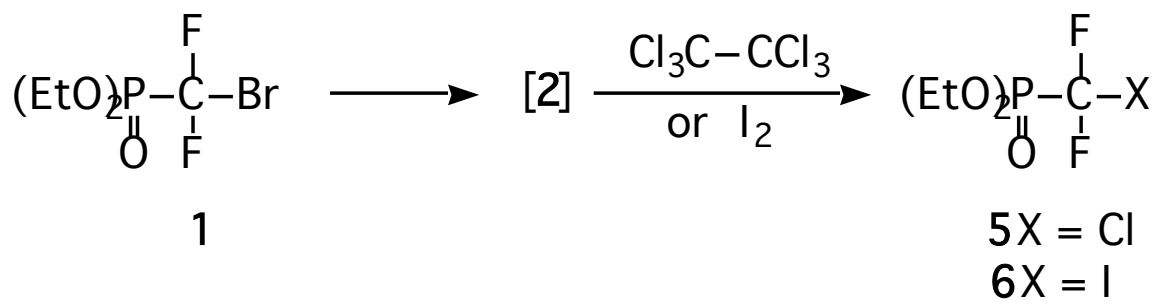

With aldehydes [10], the reaction parameters that we chose to study were stoichiometry, temperature, reaction time and effect of lithium salts. The metallation time was 5 min at $78^{\circ} \mathrm{C}$ in THF. The variation in stoichiometry 2 : aldehydes was tested for $1: 1.1$ and $1.5: 1$, the temperatures were $-78^{\circ} \mathrm{C}$ to $0^{\circ} \mathrm{C}$ for one hour and $0^{\circ} \mathrm{C}$ to room temperature for an additional hour, and in addition some of the experiments were run under the influence of electrophilic assistance by lithium bromide in order to facilitate the condensation. The composition of the crude reaction mixture was determined by ${ }^{31} \mathrm{P}$ and ${ }^{1} \mathrm{H}$ NMR spectroscopy.

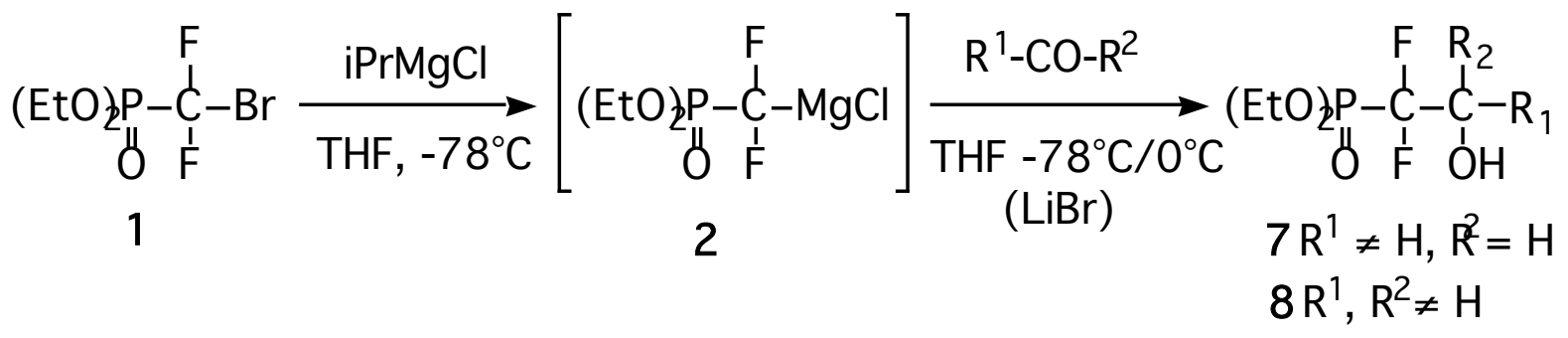

The addition of an aldehyde to $\mathbf{2}$ in stoichiometric ratio led to two phosphorus products, 7 and $\mathbf{3}$, and unreacted aldehyde detected by ${ }^{1} \mathrm{H}$ NMR. For example, the 1,1-difluoro-2hydroxyethylphosphonates $\mathbf{7 e}$ and $\mathbf{7 g}$ (Table 1) were isolated after treatment in acidic medium with respectively $70 \%$ and $60 \%$ yields. The amount of 1,1-difluoro-2-hydroxyphosphonate 7 was strongly dependant on the quantity of magnesium species 2 . The increase in the amount of 2 (1.5 equiv.), leads to a mixture of 1,1-difluoro-2-hydroxyethylphosphonate 7 with $\mathbf{3}$, the ratios of which are dependant on the reaction conditions, but without any trace of aldehyde. With these new conditions the previously prepared 1,1-difluoro-2-hydroxyethylphosphonates 7e and $7 \mathrm{~g}$ were isolated with respectively $85 \%$ and $96 \%$ yields (Table 1 ). With regard to these results, there is an increase in the yield of 7 with increasing number of equivalents of 2 . These conditions were extended to a large class of aldehydes both aliphatic, aromatic and heteroaromatic, and the results of these experiments are summarised in Table 2 . 
Increasing the time of reaction between $\mathbf{2}$ and aldehydes led to a cleaner reaction, but omitting the heating step between $0^{\circ} \mathrm{C}$ and room temperature had a negative effect since formation of a third compound $\mathbf{1 3}$ took place. This was detected by ${ }^{31} \mathrm{P}$ NMR spectroscopy ( $\delta$ $\left.{ }^{31} \mathrm{P}(\mathrm{THF})+3.4\right)$ and isolated by hydrolysing the reaction mixture at an early stage. After column chromatography, a mixture of $\mathbf{7}$ and $\mathbf{1 3}$ with $\mathbf{1 3}$ as the minor product was obtained. Trituration with hexane allowed $\mathbf{1 3}$ contaminated with a small amount of $\mathbf{7}$ to be obtained. Thus 13 was attributed to difluoromethyl bis-(diethylphosphonate) in accordance with ${ }^{19} \mathrm{~F}$ NMR spectroscopy $\left(\delta{ }^{19} \mathrm{~F}\left(\mathrm{CDCl}_{3}\right) 122.0\left(\mathrm{t},{ }^{2} J(\mathrm{P}-\mathrm{F})=87.6\right)\right)$ and mass spectrum $(\mathrm{m} / \mathrm{z}(\mathrm{IE})$ 324).

Performing the reaction between 2 and aldehydes in the presence of $\mathrm{LiBr}$, in stoichiometric ratio, had a beneficial effect since the amount of 1,1-difluoro-2-hydroxyphosphonates 7 did not decrease by running the reaction at $-40^{\circ} \mathrm{C}$ instead of $-78^{\circ} \mathrm{C}$. This is particularly obvious in the case of 4-methoxybenzaldehyde (Table 1 and Fig. 1), where the presence of $\mathrm{LiBr}$ made the formation of product $\mathbf{7 g}$ easier, whereas decomposition mainly took place at the same temperature $\left(-40^{\circ} \mathrm{C}\right)$ in the absence of $\mathrm{LiBr}$.

\begin{tabular}{cll}
\multicolumn{3}{c}{ TABLE 1. Effect of $\mathrm{LiBr}$ on the reaction of $\mathbf{2}$ with 4-methoxybenzaldehyde } \\
\hline Reaction & Yields \\
temperature & without $\mathrm{LiBr}$ & with $\mathrm{LiBr}$ \\
\hline$-78^{\circ} \mathrm{C}$ & $96 \%$ & $95 \%$ \\
$-40^{\circ} \mathrm{C}$ & partial decomposition & $95 \%$ \\
$-20^{\circ} \mathrm{C}$ & major decomposition & major decomposition \\
\hline
\end{tabular}

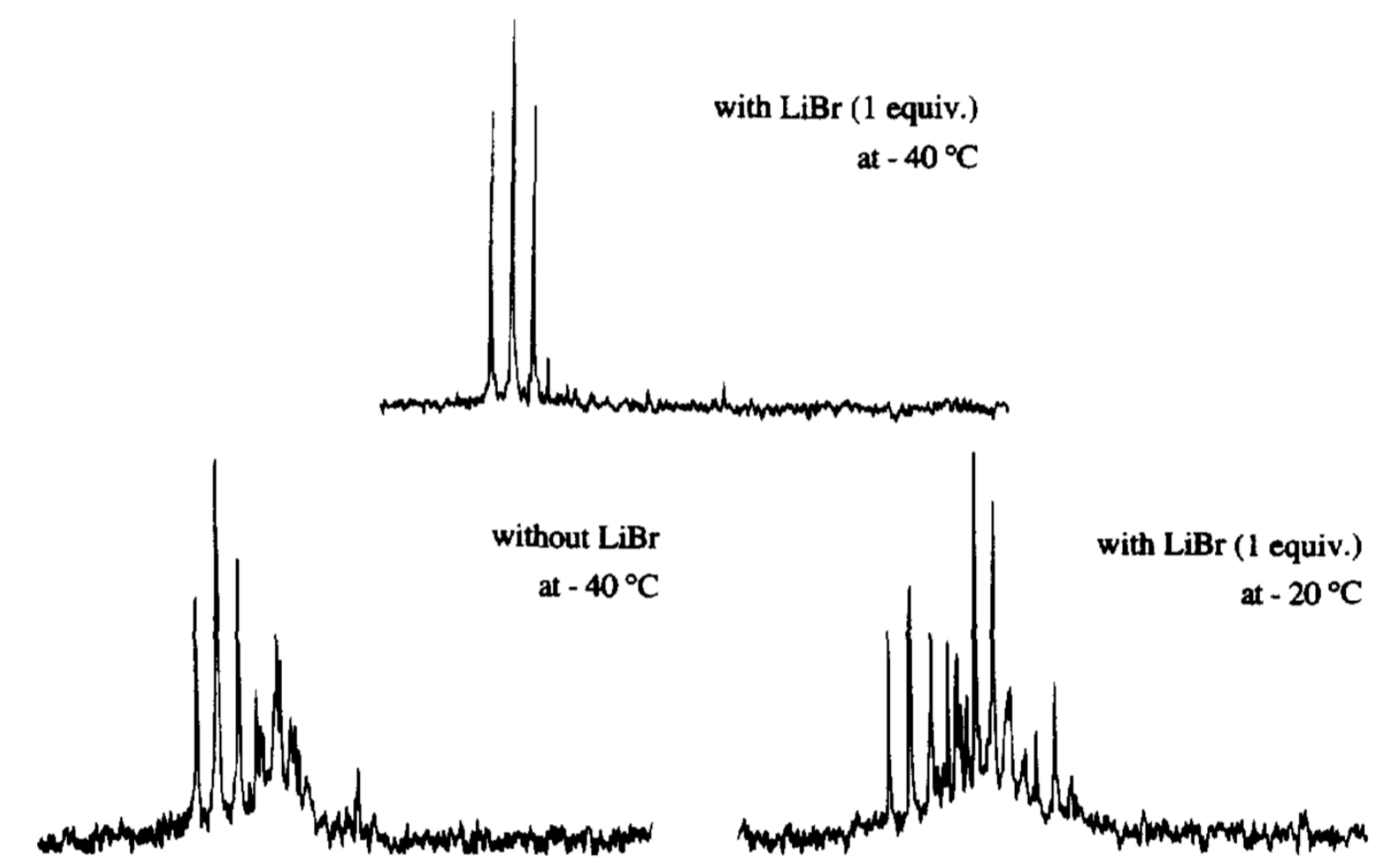

Fig. 1. ${ }^{31} \mathrm{P}$ NMR spectra of the condensation reaction of 2 with 4-methoxybenzaldehyde under various experimental conditions. 
We also examined the reactivity of ketones (Table 3). The metallation reaction was performed at $-78^{\circ} \mathrm{C}$, and the resulting magnesium species 2 was heated to $-40^{\circ} \mathrm{C}$ and reacted at this temperature with ketones in a stoichiometric ratio of $1.5: 1$ in the presence of $\mathrm{LiBr}$ (1.5 equiv.). In spite of these conditions the conversion to the 1,1-difluoro-2-hydroxyethylphosphonate $\mathbf{8}$ was never complete and the maximum yield of these reactions was $82 \%$.

\begin{tabular}{|c|c|c|}
\hline Compd 7 & $\mathrm{R}^{1}$ & Yields (\%) \\
\hline $\mathbf{a}$ & $-\left(\mathrm{CH}_{2}\right)_{6} \mathrm{CH}_{3}$ & $85^{\mathrm{b}}$ \\
\hline b & $-\mathrm{CH}\left(\mathrm{CH}_{3}\right) \mathrm{CH}_{2} \mathrm{CH}_{3}$ & $70^{\mathrm{a}^{*}}$ \\
\hline c & $-\mathrm{C}\left(\mathrm{CH}_{3}\right)_{3}$ & $84^{\mathrm{a}}$ \\
\hline d & $-\mathrm{CH}=\mathrm{CHCH}_{3}(\mathrm{E})$ & $89^{b}$ \\
\hline $\mathbf{e}$ & & $85^{\mathrm{b}}$ \\
\hline $\mathbf{f}$ & $\mathrm{CH}_{3}$ & $95^{\mathrm{b}}$ \\
\hline g & $\mathrm{OCH}_{3}$ & $96^{\mathrm{b}}$ \\
\hline $\mathbf{h}$ & $\mathrm{N}\left(\mathrm{CH}_{3}\right)_{2}$ & $92^{b}$ \\
\hline $\mathbf{i}$ & & $94^{b}$ \\
\hline $\mathbf{j}$ & & $96^{\mathrm{b}}$ \\
\hline $\mathbf{k}$ & & $82^{b}$ \\
\hline
\end{tabular}

Products are purified by distillation (a) or by chromatography (b).

* One diastereomer partially distilled with $\mathbf{3}$

TABLE 3. $(\mathrm{EtO})_{2} \mathrm{P}(\mathrm{O}) \mathrm{CF}_{2} \mathrm{C}(\mathrm{OH}) \mathrm{R}^{1} \mathrm{R}^{2} \mathbf{8}$

\begin{tabular}{|c|c|c|}
\hline Compound & $-R^{1}, R^{2}-$ & Yield (\%) \\
\hline a & $-\left(\mathrm{CH}_{2}\right)_{5}-$ & 72 \\
\hline b & $\begin{array}{c}-\left(\mathrm{CH}_{2}\right)_{2}-\mathrm{CH}-\left(\mathrm{CH}_{2}\right)^{-} \\
\left.\mathrm{I}_{(}-\mathrm{CH}_{3}\right)_{3}\end{array}$ & 82 \\
\hline c & & 46 \\
\hline d & $-\mathrm{CH}_{3}$ & 63 \\
\hline
\end{tabular}


In addition, to get further information on the reactivity of the 1,1-difluoro-2hydroxyethylphosphonates $\mathbf{7}$ and $\mathbf{8}$, we explored their reactions with various bases $(\mathrm{NaH}$, tBuOK, nBuLi and LDA). Our objective was to investigate the rearrangement reactions that they might undergo and to obtain 1,1-difluoroolefines on a preparative scale. There has been some previous work in this area and the initial observation of Obayashi et al. [4c], that lithiodifluoromethylphosphonate reacts with carbonyl compounds to produce 1,1difluoroolefines, led us to examine the related chemistry of compounds $\mathbf{7}$ and $\mathbf{8}$.

In THF at room temperature, the 1,1-difluoro-2-hydroxyethylphosphonate 7e (Table 2) on reaction with $\mathrm{NaH}$ gave only the diethyl 2,2-difluoro-1-(4'-fluoro)phenylethylphosphate 9e which was isolated in pure form with $61 \%$ yield. With tBuOK, even with a large excess, formation of 9e remained the main reaction, but other by-products could be detected. With LDA and $\mathrm{nBuLi}$ in refluxing THF, there was formation of one major product the 1,1difluoromethyl-(4'-fluoro)benzylalcohol 11e, indicating that the prior rearrangement of $\mathbf{7 e}$ into phosphate was followed by a loss of the phosphate moiety. By comparison the reaction was extended to 8a (Table 3) and we observed that rearrangement to phosphate remained the main process. Rearrangement with $\mathrm{NaH}$ giving the best results, it has been extended to several 2-hydroxyphosphonates $\mathbf{7}(\mathbf{a}, \mathbf{b}, \mathbf{c}, \mathbf{e}, \mathbf{f}, \mathbf{g}, \mathbf{h}, \mathbf{k}$, Table 2) and $\mathbf{8}(\mathbf{a}, \mathbf{c}$, Table 3) in order to obtain the phosphates $\mathbf{9}$ and $\mathbf{1 0}$ which have been isolated with the following yields : $\mathbf{9 a}$

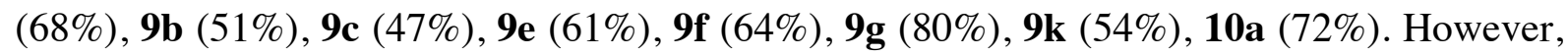
in all the reported experiments, the desired 1,1-difluoroolefine was never detected except for 7h $(15 \%)$, and $\mathbf{8 c}(20 \%)$.

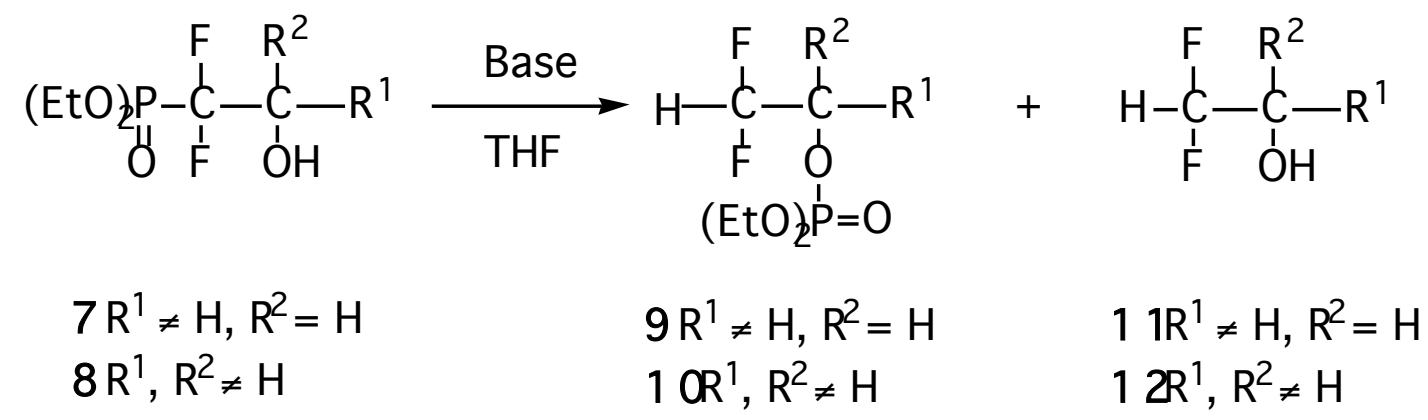

\section{Conclusion}

When bromodifluoromethylphosphonate $\mathbf{1}$ is treated with iPrMgCl in THF and the resulting magnesium species $\mathbf{2}$, reacted with an electrophile, the products formation will be influenced by stoichiometry, temperature and presence or absence of salts. The right balance between these three factors seems crucial for the synthetic applications of $\mathbf{2}$ and has led to the development of an efficient system for the formation of 1,1-difluoro-2-hydroxyethylphosphonates. However, the aim of this work was to improve the efficiency of the conditions 
leading to the formation to 1,1-difluoroolefines. The reported results indicate that no improvement was obtained by using a step by step process and no additional benefit was achieved in comparison with previous results.

\section{Experimental section}

NMR spectra were recorded on a Bruker AC 200 spectrometer operating at $200 \mathrm{MHz}$ for proton, 50.3 MHz for carbon and 81.01 MHz for phosphorus. ${ }^{31} \mathrm{P}$ downfield shifts $(\delta)$ are expressed with a positive sign, in ppm, relative to external $85 \% \mathrm{H}_{3} \mathrm{PO}_{4}$ in $\mathrm{H}_{2} \mathrm{O} .{ }^{1} \mathrm{H}$ and ${ }^{13} \mathrm{C}$ chemical shifts $(\delta)$ are reported in ppm relative to $\mathrm{CDCl}_{3}$ as internal standard. ${ }^{19} \mathrm{~F}$ NMR spectra were recorded on a Brucker AC 250 spectrometer operating at $235 \mathrm{MHz} .{ }^{19} \mathrm{~F}$ chemical shifts $(\delta)$ are expressed in ppm with a positive sign relative to $\mathrm{CFCl}_{3}$ as internal standard in $\mathrm{CDCl}_{3}$. Positive value of coupling constants $(J)$ are given in Hertz. The following abbreviations are used: s, d, t, q, p, m for singlet, doublet, triplet, quadruplet, pentuplet and multiplet respectively.

Low-resolution mass spectra were recorded on a Hewlet Packard 5989 B mass spectrometer.

Organic solvents were purified by standard procedures. THF was distilled under an inert atmosphere from purple solutions of sodium: benzophenone ketyl. The synthesis of all compounds were carried out under dry nitrogen.

\subsection{Diethyl 1,1-difluoro-1-bromomethylphosphonate 1}

A $1 \mathrm{~L}$ reactor equipped with a mechanical stirrer, thermometer, efficient reflux condenser, and an addition funnel was charged with dibromodifluoromethane (115 g, $0.55 \mathrm{~mol})$ and THF $(300 \mathrm{~mL})$ and flushed with nitrogen. Stirring was initiated and the solution was warmed by immersing the flask in an oil bath heated at $60^{\circ} \mathrm{C}$, triethyl phosphite $(83 \mathrm{~g}, 0.5 \mathrm{~mol})$ was then added dropwise over $1 \mathrm{~h}$. After an additional $30 \mathrm{~min}$ at $60^{\circ} \mathrm{C}$, the reaction mixture was cooled and the solvent was removed under reduced pressure. The crude product 1 (99\%) was purified by bulb-to-bulb distillation (b.p. $145-155^{\circ} \mathrm{C} / 0.5 \mathrm{~mm} \mathrm{Hg}$ ). Yield $=96 \%$.

${ }^{31} \mathrm{P}$ NMR $\left(\mathrm{CDCl}_{3}\right): \delta-2.6\left(\mathrm{t},{ }^{2} J(\mathrm{P}-\mathrm{F})=93\right) ;{ }^{1} \mathrm{H} \mathrm{NMR}\left(\mathrm{CDCl}_{3}\right): \delta 1.40\left(\mathrm{t}, 6 \mathrm{H},{ }^{3} J(\mathrm{H}-\mathrm{H})=\right.$ $\left.7.1, \mathrm{CH}_{3} \mathrm{CH}_{2} \mathrm{O}\right), 4.35\left(\mathrm{qd}, 4 \mathrm{H},{ }^{3} J(\mathrm{H}-\mathrm{H})=7.1,{ }^{3} J(\mathrm{H}-\mathrm{P})=8.2, \mathrm{CH}_{3} \mathrm{CH}_{2} \mathrm{O}\right) ;{ }^{13} \mathrm{C} \mathrm{NMR}$ $\left(\mathrm{CDCl}_{3}\right): \delta 17.0\left(\mathrm{~d},{ }^{3} J(\mathrm{C}-\mathrm{P})=5.8, \underline{\mathrm{CH}}_{3} \mathrm{CH}_{2} \mathrm{O}\right), 67.0\left(\mathrm{~d},{ }^{2} J(\mathrm{C}-\mathrm{P})=6.4, \mathrm{CH}_{3} \underline{\mathrm{CH}}_{2} \mathrm{O}\right), 117.3(\mathrm{td}$, $\left.{ }^{1} J(\mathrm{C}-\mathrm{F})=328.9,{ }^{1} J(\mathrm{C}-\mathrm{P})=238.2, \mathrm{PCF}_{2} \mathrm{Br}\right)$. m/z (EI) $269\left(\mathrm{M}+\mathrm{H}^{+}, 5 \%\right), 267\left(\mathrm{M}+\mathrm{H}^{+}, 5 \%\right), 137(80), 109$ (100).

\subsection{Diethyl 1,1-difluoromethylphosphonate 3}


A $500 \mathrm{~mL}$ reactor equipped with a mechanical stirrer, thermometer, reflux condenser, and an addition funnel was charged with previously standardised $\mathrm{PrMgCl}\left(29 \mathrm{~mL}\right.$ of $1.90 \mathrm{M} \mathrm{Et}_{2} \mathrm{O}$ solution, $0.055 \mathrm{~mol})$ and THF $(120 \mathrm{~mL})$. The solution was cooled to $-78^{\circ} \mathrm{C}$ and a solution of $\mathbf{1}$ (13.4 g, $0.05 \mathrm{~mol})$ in THF (50 mL) was added dropwise. The resulting mixture was stirred for $10 \mathrm{~min}$ at $-78^{\circ} \mathrm{C}$ then at this temperature a solution of EtOH $(10 \mathrm{~mL})$ in THF $(10 \mathrm{~mL})$ was added dropwise. The reaction mixture was poured into an ice-cold mixture of $\mathrm{HCl}(40 \mathrm{~mL}$ of $3 \mathrm{M}$ solution) and $\mathrm{CH}_{2} \mathrm{Cl}_{2}(50 \mathrm{~mL})$. The aqueous layer was extracted with $\mathrm{CH}_{2} \mathrm{Cl}_{2}(2 \times 50$ $\mathrm{mL})$. The extracts were dried $\left(\mathrm{MgSO}_{4}\right)$ and the solvents were removed under reduced pressure to give the crude product 3 which was purified by bulb-to-bulb distillation (b.p. 50$\left.55^{\circ} \mathrm{C} / 0.5 \mathrm{mmHg}\right)$. Yield $=85 \%$.

${ }^{31} \mathrm{P}$ NMR $\left(\mathrm{CDCl}_{3}\right): \delta+3.1\left(\mathrm{t},{ }^{2} J(\mathrm{P}-\mathrm{F})=91\right) ;{ }^{1} \mathrm{H} \mathrm{NMR}\left(\mathrm{CDCl}_{3}\right): \delta 1.36\left(\mathrm{t}, 6 \mathrm{H},{ }^{3} J(\mathrm{H}-\mathrm{H})=\right.$ $\left.7.1, \mathrm{CH}_{3} \mathrm{CH}_{2} \mathrm{O}\right), 4.26\left(\mathrm{qd}, 4 \mathrm{H},{ }^{3} J(\mathrm{H}-\mathrm{H})=7.1,{ }^{3} J(\mathrm{H}-\mathrm{P})=8.2, \mathrm{CH}_{3} \underline{\mathrm{C}}_{2} \mathrm{O}\right), 5.89\left(\mathrm{td}, 1 \mathrm{H},{ }^{2} J(\mathrm{H}-\right.$ $\left.\mathrm{F})=48.7,{ }^{2} J(\mathrm{H}-\mathrm{P})=26.9, \mathrm{PCF}_{2} \underline{\mathrm{H}}\right) ;{ }^{13} \mathrm{C} \mathrm{NMR}\left(\mathrm{CDCl}_{3}\right): \delta 16.9\left(\mathrm{~d},{ }^{3} J(\mathrm{C}-\mathrm{P})=5.7\right.$, $\left.\mathrm{CH}_{3} \mathrm{CH}_{2} \mathrm{O}\right), 65.1\left(\mathrm{~d},{ }^{2} J(\mathrm{C}-\mathrm{P})=7.0, \mathrm{CH}_{3} \underline{\mathrm{CH}}_{2} \mathrm{O}\right), 112.0\left(\mathrm{td},{ }^{1} J(\mathrm{C}-\mathrm{F})=257.9,{ }^{1} J(\mathrm{C}-\mathrm{P})=213.6\right.$, $\left.\mathrm{PCF}_{2} \mathrm{H}\right)$.

m/z (EI) $189\left(\mathrm{M}+\mathrm{H}^{+}, 1 \%\right), 137$ (60), 160 (100).

\subsection{Diethyl 1,1-difluoro-1-trimethylsilylmethylphosphonate 4}

A $500 \mathrm{~mL}$ reactor equipped as above was charged with iPrMgCl $\left(29 \mathrm{~mL}\right.$ of $1.90 \mathrm{M} \mathrm{Et}_{2} \mathrm{O}$ solution, $0.055 \mathrm{~mol})$ and $\mathrm{THF}(120 \mathrm{~mL})$. The solution was cooled to $-78^{\circ} \mathrm{C}$ and a solution of $\mathbf{1}$ $(13.4 \mathrm{~g}, 0.05 \mathrm{~mol})$ and chlorotrimethylsilane $(6 \mathrm{~g}, 0.055 \mathrm{~mol})$ in THF $(50 \mathrm{~mL})$ was added dropwise. The resulting mixture was stirred for $15 \mathrm{~min}$ at $-78^{\circ} \mathrm{C}$ then poured into an ice-cold stirred mixture of $\mathrm{HCl}\left(40 \mathrm{~mL}\right.$ of $3 \mathrm{M}$ solution) and $\mathrm{CH}_{2} \mathrm{Cl}_{2}(50 \mathrm{~mL})$. The aqueous layer was extracted with $\mathrm{CH}_{2} \mathrm{Cl}_{2}(2 \times 50 \mathrm{~mL})$. The extracts were dried $\left(\mathrm{MgSO}_{4}\right)$ and the solvents were removed under reduced pressure to give the crude product 4 which was purified by bulb-tobulb distillation (b.p. $90-95^{\circ} \mathrm{C} / 0.5 \mathrm{~mm} \mathrm{Hg}$ ). Yield $=90 \%$.

${ }^{31} \mathrm{P}$ NMR $\left(\mathrm{CDCl}_{3}\right): \delta+7.7\left(\mathrm{t},{ }^{2} J(\mathrm{P}-\mathrm{F})=92\right) ;{ }^{1} \mathrm{H} \mathrm{NMR}\left(\mathrm{CDCl}_{3}\right): \delta 0.24\left(\mathrm{~s}, 9 \mathrm{H}, \mathrm{Si}\left(\mathrm{C}_{3}\right)_{3}\right)$, $1.34\left(\mathrm{t}, 6 \mathrm{H},{ }^{3} J(\mathrm{H}-\mathrm{H})=7.1, \mathrm{C}_{3} \mathrm{CH}_{2} \mathrm{O}\right), 4.23\left(\mathrm{qd}, 4 \mathrm{H},{ }^{3} J(\mathrm{H}-\mathrm{H})=7.1,{ }^{3} J(\mathrm{H}-\mathrm{P})=7.1\right.$, $\left.\mathrm{CH}_{3} \mathrm{CH}_{2} \mathrm{O}\right) ;{ }^{13} \mathrm{C} \mathrm{NMR}\left(\mathrm{CDCl}_{3}\right): \delta-4.8\left(\mathrm{~s}, \mathrm{Si}\left(\underline{\mathrm{CH}}_{3}\right)_{3}\right), 16.2\left(\mathrm{~d},{ }^{3} J(\mathrm{C}-\mathrm{P})=5.4, \underline{\mathrm{CH}}_{3} \mathrm{CH}_{2} \mathrm{O}\right)$, $63.5\left(\mathrm{~d},{ }^{2} J(\mathrm{C}-\mathrm{P})=7.5, \quad \mathrm{CH}_{3} \underline{\mathrm{CH}}_{2} \mathrm{O}\right), 126.5 \quad\left(\mathrm{td},{ }^{1} J(\mathrm{C}-\mathrm{F})=271.5,{ }^{1} J(\mathrm{C}-\mathrm{P})=165.1\right.$, $\left.\mathrm{PCF}_{2} \mathrm{Si}\left(\mathrm{CH}_{3}\right)_{3}\right)$.

$\mathrm{m} / \mathrm{z}(\mathrm{EI}) 260\left(\mathrm{M}^{+}, 2 \%\right), 69(100)$.

\subsection{Diethyl 1,1-difluoro-1-chloromethylphosphonate 5}

A $250 \mathrm{~mL}$ reactor equipped as above was charged with iPrMgCl $\left(5.5 \mathrm{~mL}\right.$ of $2.0 \mathrm{M} \mathrm{Et}_{2} \mathrm{O}$ solution, $0.011 \mathrm{~mol})$ and THF $(10 \mathrm{~mL})$. The solution was cooled to $-78^{\circ} \mathrm{C}$ and a solution of $\mathbf{1}$ $(2.67 \mathrm{~g}, 0.01 \mathrm{~mol})$ in THF $(15 \mathrm{~mL})$ was added dropwise. The resulting mixture was stirred for 
$5 \mathrm{~min}$ at $-78^{\circ} \mathrm{C}$. Then a solution of hexachloroethane $(2.61 \mathrm{~g}, 0.011 \mathrm{~mol})$ in THF $(20 \mathrm{~mL})$ was added dropwise. The resulting mixture was stirred for $5 \mathrm{~min}$ at $-78^{\circ} \mathrm{C}$ and then allowed to warm up to $0^{\circ} \mathrm{C}$ within 2 hours. It was poured into an ice-cold mixture of $\mathrm{HCl}(20 \mathrm{~mL}$ of $3 \mathrm{M}$ solution) and $\mathrm{CH}_{2} \mathrm{Cl}_{2}(20 \mathrm{~mL})$. The aqueous layer was extracted with $\mathrm{CH}_{2} \mathrm{Cl}_{2}(2 \times 20 \mathrm{~mL})$. The extracts were dried $\left(\mathrm{MgSO}_{4}\right)$ and the solvents were removed under reduced pressure to give the crude product 5 which was purified by bulb-to-bulb distillation (b.p. $50-55^{\circ} \mathrm{C} / 0.5$ $\mathrm{mm} \mathrm{Hg}$ ). Yield $=60 \%$.

${ }^{31} \mathrm{P} \mathrm{NMR}\left(\mathrm{CDCl}_{3}\right): \delta-2.7\left(\mathrm{t},{ }^{2} J(\mathrm{P}-\mathrm{F})=101\right) ;{ }^{1} \mathrm{H} \mathrm{NMR}\left(\mathrm{CDCl}_{3}\right): \delta 1.37\left(\mathrm{t}, 6 \mathrm{H},{ }^{3} J(\mathrm{H}-\mathrm{H})=\right.$ $\left.7.1, \mathrm{CH}_{3} \mathrm{CH}_{2} \mathrm{O}\right), 4.31\left(\mathrm{p}, 4 \mathrm{H},{ }^{3} J(\mathrm{H}-\mathrm{H})=7.1,{ }^{3} J(\mathrm{H}-\mathrm{P})=7.1, \mathrm{CH}_{3} \mathrm{CH}_{2} \mathrm{O}\right) ;{ }^{13} \mathrm{C} \mathrm{NMR}\left(\mathrm{CDCl}_{3}\right)$ :

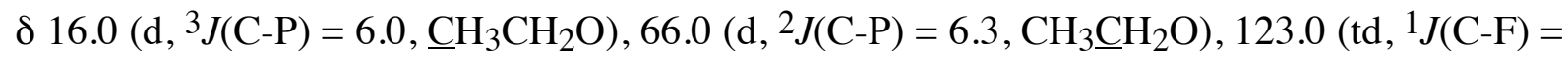
$\left.316.7,{ }^{1} J(\mathrm{C}-\mathrm{P})=249.1, \mathrm{PCF}_{2} \mathrm{Cl}\right)$.

$\mathrm{m} / \mathrm{z}(\mathrm{EI}) 223\left(\mathrm{M}^{+}, 2 \%\right), 137$ (43), 109 (100).

\subsection{Diethyl 1,1-difluoro-1-iodomethylphosphonate 6}

A $250 \mathrm{~mL}$ reactor equipped as above was charged with $\mathrm{iPrMgCl}\left(5.5 \mathrm{~mL}\right.$ of $2.0 \mathrm{M} \mathrm{Et}_{2} \mathrm{O}$ solution, $0.011 \mathrm{~mol})$ and THF (10 mL). The solution was cooled to $-78^{\circ} \mathrm{C}$ and a solution of 1 $(2.67 \mathrm{~g}, 0.01 \mathrm{~mol})$ in THF $(15 \mathrm{~mL})$ was added dropwise. The resulting mixture was stirred for $5 \mathrm{~min}$ at $-78^{\circ} \mathrm{C}$. Then a solution of iodine $(2.54 \mathrm{~g}, 0.011 \mathrm{~mol})$ in THF $(20 \mathrm{~mL})$ was added dropwise. The resulting mixture was stirred for $5 \mathrm{~min}$ at $-78^{\circ} \mathrm{C}$ and then allowed to warm up to $0^{\circ} \mathrm{C}$ within 2 hours. It was poured into an ice-cold mixture of $\mathrm{HCl}(20 \mathrm{~mL}$ of $3 \mathrm{M}$ solution) and $\mathrm{CH}_{2} \mathrm{Cl}_{2}(20 \mathrm{~mL})$. The aqueous layer was extracted with $\mathrm{CH}_{2} \mathrm{Cl}_{2}(2 \times 20 \mathrm{~mL})$. The extracts were washed with an aqueous sodium bisulfite solution, dried $\left(\mathrm{MgSO}_{4}\right)$ and the solvents were removed under reduced pressure to give the crude product $\mathbf{6}$ which was purified by bulb-tobulb distillation (b.p. $95-100^{\circ} \mathrm{C} / 0.5 \mathrm{~mm} \mathrm{Hg}$ ). Yield $=48 \%$.

${ }^{31} \mathrm{P}$ NMR $\left(\mathrm{CDCl}_{3}\right): \delta-3.8\left(\mathrm{t},{ }^{2} J(\mathrm{P}-\mathrm{F})=86\right) ;{ }^{1} \mathrm{H} \mathrm{NMR}\left(\mathrm{CDCl}_{3}\right): \delta 1.39\left(\mathrm{t}, 6 \mathrm{H},{ }^{3} J(\mathrm{H}-\mathrm{H})=\right.$ $\left.7.1, \mathrm{C}_{3} \mathrm{CH}_{2} \mathrm{O}\right), 4.34\left(\mathrm{p}, 4 \mathrm{H},{ }^{3} J(\mathrm{H}-\mathrm{H})=7.1,{ }^{3} J(\mathrm{H}-\mathrm{P})=7.1, \mathrm{CH}_{3} \mathrm{CH}_{2} \mathrm{O}\right) ;{ }^{13} \mathrm{C} \mathrm{NMR}\left(\mathrm{CDCl}_{3}\right)$ : $\delta 16.2\left(\mathrm{~d},{ }^{3} J(\mathrm{C}-\mathrm{P})=5.4, \underline{\mathrm{CH}}_{3} \mathrm{CH}_{2} \mathrm{O}\right), 66.1\left(\mathrm{~d},{ }^{2} J(\mathrm{C}-\mathrm{P})=6.4, \mathrm{CH}_{3} \underline{\mathrm{CH}_{2} \mathrm{O}}\right), 97.2\left(\mathrm{td},{ }^{1} J(\mathrm{C}-\mathrm{F})=\right.$ $\left.331.2,{ }^{1} \mathrm{~J}(\mathrm{C}-\mathrm{P})=218.1, \mathrm{PCF}_{2} \mathrm{I}\right)$.

$\mathrm{m} / \mathrm{z}$ (EI) $315\left(\mathrm{M}+\mathrm{H}^{+}, 3 \%\right), 187$ (60).

\subsection{General procedure for the condensation of 2 with aldehydes}

A $250 \mathrm{~mL}$ reactor equipped as above was charged with $\mathrm{iPrMgCl}\left(7.5 \mathrm{~mL}\right.$ of $1.90 \mathrm{M} \mathrm{Et}_{2} \mathrm{O}$ solution, $0.015 \mathrm{~mol})$ and THF (20 mL). The solution was cooled to $-78^{\circ} \mathrm{C}$ and a solution of 1 $(4.00 \mathrm{~g}, 0.015 \mathrm{~mol})$ in THF $(20 \mathrm{~mL})$ was added dropwise. The resulting mixture was stirred for $5 \mathrm{~min}$ at $-78^{\circ} \mathrm{C}$ and at this temperature a solution of aldehyde $(0.01 \mathrm{~mol})$ in THF $(20 \mathrm{~mL})$ was added dropwise. The resulting mixture was stirred for $5 \mathrm{~min}$ at $-78^{\circ} \mathrm{C}$ then was allowed to warm up to $0^{\circ} \mathrm{C}$ within one hour and from $0^{\circ} \mathrm{C}$ to room temperature for an additional hour. 
The reaction mixture* was poured into an ice-cold mixture of $\mathrm{HCl}$ (20 $\mathrm{mL}$ of $2 \mathrm{M}$ solution) and $\mathrm{CH}_{2} \mathrm{Cl}_{2}(20 \mathrm{~mL})$. The aqueous layer was extracted with $\mathrm{CH}_{2} \mathrm{Cl}_{2}(2 \times 20 \mathrm{~mL})$. The extracts were dried $\left(\mathrm{MgSO}_{4}\right)$ and the solvents were removed under reduced pressure to give the crude product 7 mixed with 3 which was prior eliminated by heating the crude at $70{ }^{\circ} \mathrm{C}$ under 0.5 $\mathrm{mm} \mathrm{Hg}$ for one hour. Then 7 was purified either by bulb-to-bulb distillation or by column chromatography (see Table 2).

* Work-up were performed in a different manner for the 2-(4'-dimethylamino)phenyl- (7h) and the 2-2'-pyridyl- (7k) phosphonates : Ice-cold $\mathrm{HCl}$ (7.5 mL of $2 \mathrm{M}$ solution, $0.015 \mathrm{~mol})$ was rapidly added to the reaction mixture of $\mathbf{7 h}$ before extraction ; whereas the reaction mixture of $\mathbf{7 k}$ was poured into an ice-cold mixture of saturated ammonium salt solution (20 $\mathrm{mL})$ and $\mathrm{CH}_{2} \mathrm{Cl}_{2}(20 \mathrm{~mL})$.

\subsubsection{Diethyl 1,1-difluoro-2-heptyl-2-hydroxyethylphosphonate 7a}

${ }^{31} \mathrm{P} \mathrm{NMR}\left(\mathrm{CDCl}_{3}\right): \delta+5.4\left(\mathrm{t},{ }^{2} J(\mathrm{P}-\mathrm{F})=104\right) ;{ }^{19} \mathrm{~F} \mathrm{NMR}\left(\mathrm{CDCl}_{3}\right): \delta-116.8\left(\mathrm{ddd},{ }^{2} J(\mathrm{~F}-\mathrm{F})=\right.$ $\left.304.7,{ }^{2} J(\mathrm{~F}-\mathrm{P})=102.8,{ }^{3} J(\mathrm{~F}-\mathrm{H})=7.6, \mathrm{PCF}_{\mathrm{A}} \mathrm{F}_{\mathrm{B}} \mathrm{CHOH}\right),-125.6\left(\mathrm{ddd},{ }^{2} J(\mathrm{~F}-\mathrm{F})=304.7,{ }^{2} J(\mathrm{~F}-\right.$ $\left.\mathrm{P})=106.6,{ }^{3} J(\mathrm{~F}-\mathrm{H})=19.0, \mathrm{PCF}_{\mathrm{A}} \underline{\mathrm{F}}_{\mathrm{B}} \mathrm{CHOH}\right) ;{ }^{1} \mathrm{H} \mathrm{NMR}\left(\mathrm{CDCl}_{3}\right): \delta 0.87\left(\mathrm{t}, 3 \mathrm{H},{ }^{3} J(\mathrm{H}-\mathrm{H})=\right.$ 6.3, $\left.\left(\mathrm{CH}_{2}\right)_{6} \mathrm{CH}_{3}\right), 1.29\left(\mathrm{~s}, 8 \mathrm{H},\left(\mathrm{CH}_{2}\right)_{4} \mathrm{CH}_{3}\right), 1.38\left(\mathrm{t}, 6 \mathrm{H},{ }^{3} \mathrm{~J}(\mathrm{H}-\mathrm{H})=7.1, \mathrm{CH}_{3} \mathrm{CH}_{2} \mathrm{O}\right), 1.64(\mathrm{~m}$, $\left.4 \mathrm{H}, \mathrm{CHOH}\left(\mathrm{C}_{2}\right)_{2}\right), 3.4\left(\mathrm{~m}, 1 \mathrm{H}, \mathrm{PCF}_{2} \mathrm{CHO} \underline{H}\right), 3.9\left(\mathrm{~m}, 1 \mathrm{H}, \mathrm{PCF}_{2} \mathrm{C} \underline{\mathrm{HOH}}\right), 4.29\left(\mathrm{p}, 4 \mathrm{H},{ }^{3} \mathrm{~J}(\mathrm{H}-\right.$ $\left.\mathrm{H})={ }^{3} J(\mathrm{H}-\mathrm{P})=7.1, \mathrm{CH}_{3} \mathrm{CH}_{2} \mathrm{O}\right) ;{ }^{13} \mathrm{C} \mathrm{NMR}\left(\mathrm{CDCl}_{3}\right): \delta 14.0\left(\mathrm{~s},\left(\mathrm{CH}_{2}\right)_{6} \mathrm{CH}_{3}\right), 16.3\left(\mathrm{~d},{ }^{3} J(\mathrm{C}-\right.$ $\left.\mathrm{P})=5.5, \underline{\mathrm{CH}}_{3} \mathrm{CH}_{2} \mathrm{O}\right), 22.7\left(\mathrm{~s}, \underline{\mathrm{CH}}_{2}\right), 25.4\left(\mathrm{~s}, \underline{\mathrm{CH}}_{2}\right), 28.9\left(\mathrm{~s}, \underline{\mathrm{C}} \mathrm{H}_{2}\right), 29.4\left(\mathrm{~d},{ }^{3} J(\mathrm{C}-\mathrm{P})=7.5\right.$, $\left.\mathrm{CHOHCH}_{2}\right), 29.8\left(\mathrm{~s}, \underline{\mathrm{CH}}_{2}\right), 31.9\left(\mathrm{~s}, \underline{\mathrm{CH}}_{2}\right), 64.6\left(\mathrm{~s}, \mathrm{CH}_{3} \underline{\mathrm{CH}}_{2} \mathrm{O}\right), 71.2$ (m, X part of ABMX system, ${ }^{2} J \approx 23.3$, PMCFAFBCXHOH), 119.7 (ddd, X part of ABMX system, ${ }^{1} J(\mathrm{C}-\mathrm{F})=$ 268.6, ${ }^{1} J(\mathrm{C}-\mathrm{F})=264.0,{ }^{1} J(\mathrm{C}-\mathrm{P})=207.6, \mathrm{PMCXF}_{\mathrm{A}} \mathrm{FBCHOH}_{\mathrm{B}}$.

$\mathrm{m} / \mathrm{z}(\mathrm{EI}) 317\left(\mathrm{M}+\mathrm{H}^{+}, 6 \%\right), 188(100), 161$ (95).

\subsubsection{Diethyl 1,1-difluoro-2-(2'-butyl)-2-hydroxyethylphosphonate (two diastereomers) $7 \boldsymbol{b}$}

${ }^{31} \mathrm{P}$ NMR $\left(\mathrm{CDCl}_{3}\right): \delta+5.5\left(\mathrm{t},{ }^{2} J(\mathrm{P}-\mathrm{F})=105, \mathrm{P}_{\text {maj. }}\right),+5.0\left(\mathrm{dd},{ }^{2} J(\mathrm{P}-\mathrm{F})=99\right.$ and $\left.105, \mathrm{P}_{\text {min. }}\right)$ ; ${ }^{1} \mathrm{H}$ NMR $\left(\mathrm{CDCl}_{3}\right): \delta 0.89\left(\mathrm{t}, 3 \mathrm{H},{ }^{3} J(\mathrm{H}-\mathrm{H})=7.4, \mathrm{CH}_{2} \mathrm{CH}_{3}\right), 0.98\left(\mathrm{~d}, 3 \mathrm{H},{ }^{3} J(\mathrm{H}-\mathrm{H})=6.7\right.$, $\left.\mathrm{CHCH}_{3}\right), 1.35\left(\mathrm{t}, 6 \mathrm{H},{ }^{3} \mathrm{~J}(\mathrm{H}-\mathrm{H})=7.1, \mathrm{CH}_{3} \mathrm{CH}_{2} \mathrm{O}\right), 1.5\left(\mathrm{~m}, 2 \mathrm{H}, \mathrm{CH}_{2} \mathrm{CH}_{3}\right), 1.9(\mathrm{~m}, 1 \mathrm{H}, \mathrm{CHOH}-$ $\mathrm{C} \underline{\mathrm{H}}), 3.5\left(\mathrm{~m}, 1 \mathrm{H}, \mathrm{PCF}_{2} \mathrm{CHO} \underline{\mathrm{H}}\right), 3.7\left(\mathrm{~m}, 1 \mathrm{H}, \mathrm{PCF}_{2} \mathrm{C} \underline{\mathrm{HOH}}\right), 4.25\left(\mathrm{~m}, 4 \mathrm{H},{ }^{3} J(\mathrm{H}-\mathrm{H})=7.1\right.$, $\mathrm{CH}_{3} \mathrm{CH}_{2} \mathrm{O}$ ) ; ${ }^{13} \mathrm{C} \mathrm{NMR}\left(\mathrm{CDCl}_{3}\right.$ ): $\delta 11.7$ (s, $\mathrm{CH}_{2} \underline{\mathrm{CH}}_{3}$ min.), 12.3 (s, $\mathrm{CH}_{2} \underline{\mathrm{CH}}_{3}$ maj.), 14.1 (s, $\mathrm{CH} \underline{C H}_{3}$ maj.), 16.3 (s, $\mathrm{CH} \mathrm{H}_{3}$ min.), 17.1 (d, $\left.{ }^{3} J(\mathrm{C}-\mathrm{P})=5.3, \underline{\mathrm{CH}_{3}} \mathrm{CH}_{2} \mathrm{O}\right), 24.7$ (s, $\underline{\mathrm{CH}}_{2} \mathrm{CH}_{3}$ min.), 27.8 (s, $\underline{\mathrm{CH}}_{2} \mathrm{CH}_{3}$ maj.), 34.9 (s, $\mathrm{CHOH}-\underline{\mathrm{CH}}_{\text {maj. }}$ ), 35.7 (s, $\mathrm{CHOH}-\mathrm{CH}_{\text {min.) }}$ ), 65.5 (s, $\mathrm{CH}_{3} \underline{\mathrm{CH}}_{2} \mathrm{O}$ ), 73.3 (m, $\underline{\mathrm{C}} \mathrm{HOH}_{\text {maj. }}$ ), 75.3 (m, $\underline{\mathrm{CHOH}}_{\text {min. }}$ ), 120.9 (ddd, X part of ABMX system, ${ }^{1} J(\mathrm{C}-\mathrm{F})=270.6,{ }^{1} J(\mathrm{C}-\mathrm{F})=265.4,{ }^{1} J(\mathrm{C}-\mathrm{P})=208.4, \mathrm{PMCXF}_{\mathrm{CAFBCHOH}}{ }_{\text {maj. }}$ ), $121.4(\mathrm{ddd}, \mathrm{X}$ part of $\mathrm{ABMX}$ system, ${ }^{1} J(\mathrm{C}-\mathrm{F})={ }^{1} J \approx 272.2, \quad{ }^{1} J(\mathrm{C}-\mathrm{F})=266.0, \quad{ }^{1} J(\mathrm{C}-\mathrm{P})=210.0$, $\mathrm{PM}_{\mathrm{CXF}} \mathrm{FF}_{\mathrm{B}} \mathrm{HOH}_{\min }$.). 
m/z (EI) $274\left(\mathrm{M}^{+}, 2 \%\right), 217$ (46), 188 (61), 161 (100).

4.6.3. Diethyl 1,1-difluoro-2-tertbutyl-2-hydroxyethylphosphonate 7c

${ }^{31} \mathrm{P} \mathrm{NMR}\left(\mathrm{CDCl}_{3}\right): \delta+5.8\left(\mathrm{t},{ }^{2} J(\mathrm{P}-\mathrm{F})=104\right) ;{ }^{1} \mathrm{H} \mathrm{NMR}\left(\mathrm{CDCl}_{3}\right): \delta 1.05(\mathrm{~d}, 9 \mathrm{H}, J=0.9$, $\left.\mathrm{C}\left(\mathrm{CH}_{3}\right)_{3}\right), 1.36\left(\mathrm{t}, 6 \mathrm{H},{ }^{3} \mathrm{~J}(\mathrm{H}-\mathrm{H})=7.1, \mathrm{CH}_{3} \mathrm{CH}_{2} \mathrm{O}\right), 3.5\left(\mathrm{~m}, 1 \mathrm{H}, \mathrm{PCF}_{2} \mathrm{CHO} \underline{\mathrm{H}}\right), 3.71(\mathrm{ddd}, 1 \mathrm{H}$, $\mathrm{X}$ part of ABMX system, $\left.{ }^{3} J(\mathrm{C}-\mathrm{P})=28.5,{ }^{3} J(\mathrm{C}-\mathrm{F})=3.7,{ }^{3} J(\mathrm{C}-\mathrm{F})=1.4, \mathrm{PMCF}_{\mathrm{AFBCH}} \mathrm{XOH}\right)$, $4.27\left(\mathrm{qd}, 4 \mathrm{H},{ }^{3} J(\mathrm{H}-\mathrm{P})=12.0,{ }^{3} J(\mathrm{H}-\mathrm{H})=7.1, \mathrm{CH}_{3} \mathrm{CH}_{2} \mathrm{O}\right) ;{ }^{13} \mathrm{C} \mathrm{NMR}\left(\mathrm{CDCl}_{3}\right): \delta 17.0(\mathrm{~d}$, $\left.{ }^{3} J(\mathrm{C}-\mathrm{P})=5.7, \underline{\mathrm{CH}}_{3} \mathrm{CH}_{2} \mathrm{O}\right), 27.3\left(\mathrm{~s}, \mathrm{C}\left(\underline{\mathrm{CH}}_{3}\right)_{3}\right), 36.0\left(\mathrm{~d}, \underline{\mathrm{C}}\left(\mathrm{CH}_{3}\right)_{3}\right), 65.2\left(\mathrm{~d},{ }^{2} J(\mathrm{C}-\mathrm{P})=7.0\right.$, $\left.\mathrm{CH}_{3} \underline{\mathrm{CH}_{2} \mathrm{O}}\right), 65.5\left(\mathrm{~d},{ }^{2} J(\mathrm{C}-\mathrm{P})=6.6, \mathrm{CH}_{3} \mathrm{CH}_{2} \mathrm{O}\right), 76.9\left(\mathrm{~m}, \mathrm{X}\right.$ part of ABMX system, ${ }^{2} J(\mathrm{C}-\mathrm{F})=$ $\left.24.9,{ }^{2} J(\mathrm{C}-\mathrm{F})=20.8,{ }^{2} J(\mathrm{C}-\mathrm{P})=10.0, \mathrm{PMCF}_{\mathrm{AFB}} \mathrm{CXHOH}\right), 122.9(\mathrm{~m}, \mathrm{X}$ part of $\mathrm{ABMX}$ system, ${ }^{1} J(\mathrm{C}-\mathrm{F})=277.0,{ }^{1} J(\mathrm{C}-\mathrm{F})=267.4,{ }^{1} J(\mathrm{C}-\mathrm{P})=209.2, \mathrm{PMCX}_{\mathrm{MA}} \mathrm{FBCHOH}_{\mathrm{B}}$.

m/z (EI) 275 (M+H+, $100 \%), 188$ (19), 161 (56), 132 (39).

\subsubsection{Diethyl 1,1-difluoro-2-(1'-propenyl)-2-hydroxyethylphosphonate 7d}

${ }^{31} \mathrm{P}$ NMR $\left(\mathrm{CDCl}_{3}\right): \delta+5.0\left(\mathrm{t},{ }^{2} J(\mathrm{P}-\mathrm{F})=103\right) ;{ }^{19} \mathrm{~F} \mathrm{NMR}\left(\mathrm{CDCl}_{3}\right): \delta-116.4\left(\mathrm{ddd},{ }^{2} J(\mathrm{~F}-\mathrm{F})=\right.$ $\left.304.7,{ }^{2} J(\mathrm{~F}-\mathrm{P})=99.0,{ }^{3} J(\mathrm{~F}-\mathrm{H})=7.6, \mathrm{PC}_{\mathrm{A}} \mathrm{F}_{\mathrm{B}} \mathrm{CHOH}\right),-124.3\left(\mathrm{ddd},{ }^{2} J(\mathrm{~F}-\mathrm{F})=304.7,{ }^{2} J(\mathrm{~F}-\right.$ $\left.\mathrm{P})=102.8,{ }^{3} J(\mathrm{~F}-\mathrm{H})=19.0, \mathrm{PCF}_{\mathrm{A}} \mathrm{F}_{\mathrm{B}} \mathrm{CHOH}\right) ;{ }^{1} \mathrm{H} \mathrm{NMR}\left(\mathrm{CDCl}_{3}\right): \delta 1.38\left(\mathrm{t}, 6 \mathrm{H},{ }^{3} J(\mathrm{H}-\mathrm{H})=\right.$ 7.1, $\left.\mathrm{C}_{3} \mathrm{CH}_{2} \mathrm{O}\right), 1.77\left(\mathrm{~d}, 3 \mathrm{H},{ }^{3} J(\mathrm{H}-\mathrm{H})=6.7,=\mathrm{CHCH}_{3}\right), 3.20\left(\mathrm{~d}_{\text {large }}, 1 \mathrm{H},{ }^{3} J(\mathrm{H}-\mathrm{H})=3.8\right.$, $\left.\mathrm{PCF}_{2} \mathrm{CHO} \underline{\mathrm{H}}\right), 4.28\left(\mathrm{p}, 4 \mathrm{H},{ }^{3} J(\mathrm{H}-\mathrm{P})={ }^{3} J(\mathrm{H}-\mathrm{H})=7.1, \mathrm{CH}_{3} \mathrm{CH}_{2} \mathrm{O}\right), 4.4\left(\mathrm{~m}, 1 \mathrm{H}, \mathrm{PCF}_{2} \mathrm{CHOH}\right)$, $5.59\left(\mathrm{ddq}, 1 \mathrm{H},{ }^{3} J(\mathrm{H}-\mathrm{H})_{\text {trans }}=15.4,{ }^{3} J(\mathrm{H}-\mathrm{H})=6.5,{ }^{4} J(\mathrm{H}-\mathrm{H})=1.5, \mathrm{C} \underline{\mathrm{H}}=\mathrm{CHCH}_{3}\right), 5.95(\mathrm{dqd}, 1 \mathrm{H}$, $\left.{ }^{3} J(\mathrm{H}-\mathrm{H})_{\text {trans }}=15.4,{ }^{3} J(\mathrm{H}-\mathrm{H})=6.7,{ }^{4} J(\mathrm{H}-\mathrm{H})=1.0, \mathrm{CH}=\mathrm{CHCH}_{3}\right) ;{ }^{13} \mathrm{C} \mathrm{NMR}\left(\mathrm{CDCl}_{3}\right): \delta 16.3(\mathrm{~d}$, $\left.{ }^{3} J(\mathrm{C}-\mathrm{P})=5.2, \underline{\mathrm{CH}}_{3} \mathrm{CH}_{2} \mathrm{O}\right), 17.9\left(\mathrm{~s},=\mathrm{CH}^{\mathrm{CH}}{ }_{3}\right), 64.7\left(\mathrm{~d},{ }^{2} J(\mathrm{C}-\mathrm{P})=7.1, \mathrm{CH}_{3} \underline{\mathrm{CH}}_{2} \mathrm{O}\right), 72.5$ (ddd, $\mathrm{X}$ part of ABMX system, ${ }^{2} J(\mathrm{C}-\mathrm{F})=25.8, \quad{ }^{2} J(\mathrm{C}-\mathrm{F})=22.8, \quad{ }^{2} J(\mathrm{C}-\mathrm{P})=15.1$, PMCFAFBCXHOH), $118.9\left(\mathrm{ddd}, \mathrm{X}\right.$ part of $\mathrm{ABMX}$ system, ${ }^{1} J(\mathrm{C}-\mathrm{F})=269.5,{ }^{1} J(\mathrm{C}-\mathrm{F})=$ 264.2, $\left.{ }^{1} J(\mathrm{C}-\mathrm{P})=207.8, \mathrm{PM}_{\mathrm{MX}} \mathrm{FAFBCHOH}_{\mathrm{B}}\right), 125.0\left(\mathrm{~d},{ }^{3} J(\mathrm{C}-\mathrm{P})=2.3, \underline{\mathrm{CH}}=\mathrm{CHCH}_{3}\right), 132.0$ (s, $\left.\mathrm{CH}=\underline{\mathrm{C}} \mathrm{HCH}{ }_{3}\right)$.

$\mathrm{m} / \mathrm{z}(\mathrm{CI}+\mathrm{ve}) 259\left(\mathrm{M}+\mathrm{H}^{+}, 100 \%\right)$.

\subsubsection{Diethyl 1,1-difluoro-2-(4'-fluorophenyl)-2-hydroxyethylphosphonate 7e}

${ }^{31} \mathrm{P}$ NMR $\left(\mathrm{CDCl}_{3}\right): \delta+4.9\left(\mathrm{t},{ }^{2} J(\mathrm{P}-\mathrm{F})=99\right) ;{ }^{19} \mathrm{~F} \mathrm{NMR}\left(\mathrm{CDCl}_{3}\right): \delta-113.7\left(\mathrm{~m}, \mathrm{C}_{6} \mathrm{H}_{4} \mathrm{~F}\right),-$ $115.0\left(\mathrm{dd},{ }^{2} J(\mathrm{~F}-\mathrm{F})=304.7,{ }^{2} J(\mathrm{~F}-\mathrm{P})=99.0, \mathrm{PCF}_{\mathrm{A}} \mathrm{F}_{\mathrm{B}} \mathrm{CHOH}\right),-125.8\left(\mathrm{ddd},{ }^{2} J(\mathrm{~F}-\mathrm{F})=304.7\right.$, $\left.{ }^{2} J(\mathrm{~F}-\mathrm{P})=102.8,{ }^{3} J(\mathrm{~F}-\mathrm{H})=19.0, \mathrm{PCF}_{\mathrm{A}} \underline{\mathrm{F}}_{\mathrm{B}} \mathrm{CHOH}\right) ;{ }^{1} \mathrm{H} \mathrm{NMR}\left(\mathrm{CDCl}_{3}\right): \delta 1.34\left(2 \mathrm{t}, 6 \mathrm{H},{ }^{3} J(\mathrm{H}-\right.$ $\mathrm{H})=7.0,\left(\mathrm{CH}_{3} \mathrm{CH}_{2} \mathrm{O}\right)_{\mathrm{A}}$ and $\left.\mathrm{B}\right), 4.0\left(\mathrm{~m}, 1 \mathrm{H}, \mathrm{PCF}_{2} \mathrm{CHOHC}_{6} \mathrm{H}_{4} \mathrm{~F}\right), 4.24\left(\mathrm{qd}, 4 \mathrm{H},{ }^{3} J(\mathrm{H}-\mathrm{P})=8.1\right.$, ${ }^{3} J(\mathrm{H}-\mathrm{H})=7.0,\left(\mathrm{CH}_{3} \underline{\mathrm{C}}_{2} \mathrm{O}\right) \mathrm{A}$ and B $), 5.10\left(\mathrm{ddd}, 1 \mathrm{H},{ }^{3} J(\mathrm{H}-\mathrm{P})=20.4,{ }^{3} J(\mathrm{H}-\mathrm{F})=5.9,{ }^{3} J(\mathrm{H}-\mathrm{F})=\right.$ 3.9, $\left.\mathrm{PCF}_{2} \mathrm{CHOHC}_{6} \mathrm{H}_{4} \mathrm{~F}\right), 7.07\left(\mathrm{t}, 2 \mathrm{H},{ }^{3} J(\mathrm{H}-\mathrm{F})={ }^{3} J(\mathrm{H}-\mathrm{H})=8.7, \mathrm{H}_{\text {meta }}\right.$ of $\left.\mathrm{C}_{6} \mathrm{H}_{4} \mathrm{~F}\right), 7.46(\mathrm{dd}$, $2 \mathrm{H},{ }^{3} J(\mathrm{H}-\mathrm{H})=8.4,{ }^{4} J(\mathrm{H}-\mathrm{F})=8.5, \mathrm{H}_{\mathrm{ortho}}$ of $\left.\mathrm{C}_{6} \mathrm{H}_{4} \mathrm{~F}\right) ;{ }^{13} \mathrm{C} \mathrm{NMR}\left(\mathrm{CDCl}_{3}\right): \delta 17.0\left(\mathrm{~d},{ }^{3} J(\mathrm{C}-\right.$ $\left.\mathrm{P})=5.7, \underline{\mathrm{CH}}_{3} \mathrm{CH}_{2} \mathrm{O}\right), 65.7\left(\mathrm{~d},{ }^{2} J(\mathrm{C}-\mathrm{P})=6.8, \mathrm{CH}_{3} \underline{\mathrm{CH}}_{2} \mathrm{O}\right), 73.3(\mathrm{~m} 8$ peaks, $\mathrm{X}$ part of ABMX 
system, $\left.{ }^{2} J(\mathrm{C}-\mathrm{F})=26.4,{ }^{2} J(\mathrm{C}-\mathrm{F})=21.6,{ }^{2} J(\mathrm{C}-\mathrm{P})=14.4, \mathrm{PMCF}_{\mathrm{A}} \mathrm{FBCXHOH}\right), 115.7\left(\mathrm{~d},{ }^{2} J(\mathrm{C}-\right.$ $\mathrm{F})=22.0, \underline{\mathrm{C}}_{\text {meta }}$ of $\left.\mathrm{C}_{6} \mathrm{H}_{4} \mathrm{~F}\right), 118.6\left(\mathrm{ddd}, \mathrm{X}\right.$ part of $\mathrm{ABMX}$ system, ${ }^{1} \mathrm{~J}(\mathrm{C}-\mathrm{F})=271.9,{ }^{1} \mathrm{~J}(\mathrm{C}-\mathrm{F})=$ 264.9, $\left.{ }^{1} J(\mathrm{C}-\mathrm{P})=206.2, \mathrm{PMCX}_{\mathrm{M}} \mathrm{FF}_{\mathrm{B}} \mathrm{CHOH}\right), 130.6\left(\mathrm{~d},{ }^{3} J(\mathrm{C}-\mathrm{F})=8.1, \underline{\mathrm{C}}_{\text {ortho }}\right.$ of $\left.\mathrm{C}_{6} \mathrm{H}_{4} \mathrm{~F}\right)$, $131.5\left(\mathrm{t},{ }^{3} J(\mathrm{C}-\mathrm{F})=3.0, \underline{\mathrm{C}}_{\mathrm{ipso}}\right.$ of $\left.\mathrm{C}_{6} \mathrm{H}_{4} \mathrm{~F}\right), 163.7\left(\mathrm{~d},{ }^{1} J(\mathrm{C}-\mathrm{F})=249.6, \underline{\mathrm{C}}_{\text {para }}\right.$ of $\left.\mathrm{C}_{6} \mathrm{H}_{4} \mathrm{~F}\right)$.

m/z (EI) $312\left(\mathrm{M}^{+}, 1 \%\right), 188(33), 161$ (41), 132 (100).

\subsubsection{Diethyl 1,1-difluoro-2-(4'-methylphenyl)-2-hydroxyethylphosphonate $7 f$}

${ }^{31} \mathrm{P}$ NMR $\left(\mathrm{CDCl}_{3}\right): \delta+5.2\left(\mathrm{t},{ }^{2} J(\mathrm{P}-\mathrm{F})=103\right) ;{ }^{19} \mathrm{~F} \mathrm{NMR}\left(\mathrm{CDCl}_{3}\right): \delta-114.8\left(\mathrm{ddd},{ }^{2} J(\mathrm{~F}-\mathrm{F})=\right.$ $\left.304.7,{ }^{2} J(\mathrm{~F}-\mathrm{P})=99.0,{ }^{3} J(\mathrm{~F}-\mathrm{H})=7.6, \mathrm{PCF}_{\mathrm{A}} \mathrm{F}_{\mathrm{B}} \mathrm{CHOH}\right),-125.6\left(\mathrm{ddd},{ }^{2} J(\mathrm{~F}-\mathrm{F})=304.7,{ }^{2} J(\mathrm{~F}-\right.$ $\left.\mathrm{P})=106.6,{ }^{3} J(\mathrm{~F}-\mathrm{H})=19.0, \mathrm{PCF}_{\mathrm{A}} \underline{\mathrm{F}}_{\mathrm{B}} \mathrm{CHOH}\right) ;{ }^{1} \mathrm{H} \mathrm{NMR}\left(\mathrm{CDCl}_{3}\right): \delta 1.31\left(\mathrm{~m}, 6 \mathrm{H}, \mathrm{C}_{3} \mathrm{CH}_{2} \mathrm{O}\right)$, 2.35 (s, 3H, $\mathrm{C}_{6} \mathrm{H}_{4} \mathrm{CH}_{3}$ ), 4.1 (m masked $1 \mathrm{H}, \mathrm{PCF}_{2} \mathrm{CHOH}$ ), 4.23 (m, 4H, $\mathrm{CH}_{3} \mathrm{CH}_{2} \mathrm{O}$ ), 5.07 (ddd, $1 \mathrm{H},{ }^{3} J(\mathrm{H}-\mathrm{P})=20.5,{ }^{3} J(\mathrm{H}-\mathrm{F})=6.0$ et $\left.3.8, \mathrm{PCF}_{2} \mathrm{CHOH}\right), 7.18\left(\mathrm{~d}, 2 \mathrm{H},{ }^{3} J(\mathrm{H}-\mathrm{H})=8.0, \mathrm{H}_{\text {meta }}\right.$ of

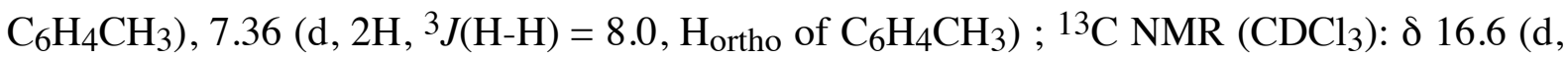

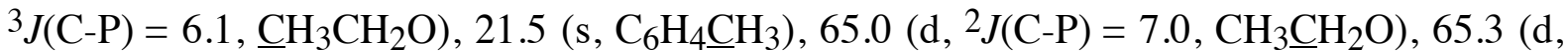
$\left.{ }^{2} J(\mathrm{C}-\mathrm{P})=6.8, \mathrm{CH}_{3} \mathrm{CH}_{2} \mathrm{O}\right), 73.3\left(\mathrm{ddd}, \mathrm{X}\right.$ part of $\mathrm{ABMX}$ system, ${ }^{2} J(\mathrm{C}-\mathrm{F})=26.5,{ }^{2} J(\mathrm{C}-\mathrm{F})=$ 21.3, $\left.{ }^{2} J(\mathrm{C}-\mathrm{P})=14.7, \mathrm{PMCF}_{\mathrm{AFBC}} \mathrm{XHOH}\right), 118.8\left(\mathrm{ddd}, \mathrm{X}\right.$ part of $\mathrm{ABMX}$ system, ${ }^{1} J(\mathrm{C}-\mathrm{F})=$ $\left.272.3,{ }^{1} \mathrm{~J}(\mathrm{C}-\mathrm{F})=263.2,{ }^{1} \mathrm{~J}(\mathrm{C}-\mathrm{P})=207.4, \mathrm{PMCXF}_{\mathrm{A}} \mathrm{FBCHOH}_{\mathrm{B}}\right), 128.5\left(\mathrm{~s}, \underline{\mathrm{C}}_{\text {meta }}\right.$ of $\left.\mathrm{C}_{6} \mathrm{H}_{4} \mathrm{CH}_{3}\right)$, 129.1 (s, $\underline{\mathrm{C}}_{\text {ortho }}$ of $\left.\mathrm{C}_{6} \mathrm{H}_{4} \mathrm{CH}_{3}\right), 133.1\left(\mathrm{~d},{ }^{3} J(\mathrm{C}-\mathrm{P})=5.7, \underline{\mathrm{C}}_{\mathrm{ipso}}\right.$ of $\left.\mathrm{C}_{6} \mathrm{H}_{4} \mathrm{CH}_{3}\right), 138.7$ (s, $\underline{\mathrm{C}}_{\text {para }}$ of $\left.\mathrm{C}_{6} \mathrm{H}_{4} \mathrm{CH}_{3}\right)$.

$\mathrm{m} / \mathrm{z}(\mathrm{EI}) 308\left(\mathrm{M}^{+}, 7 \%\right), 188(48), 161(55), 132(100)$.

\subsubsection{Diethyl 1,1-difluoro-2-(4'-methoxyphenyl)-2-hydroxyethylphosphonate $\mathbf{7 g}$}

${ }^{31} \mathrm{P}$ NMR $\left(\mathrm{CDCl}_{3}\right): \delta+5.2\left(\mathrm{t},{ }^{2} J(\mathrm{P}-\mathrm{F})=103\right) ;{ }^{19} \mathrm{~F}$ NMR $\left(\mathrm{CDCl}_{3}\right): \delta-115.2\left(\mathrm{dd},{ }^{2} J(\mathrm{~F}-\mathrm{F})=\right.$ $\left.304.7,{ }^{2} J(\mathrm{~F}-\mathrm{P})=99.0, \mathrm{PCF}_{\mathrm{A}} \mathrm{F}_{\mathrm{B}} \mathrm{CHOH}\right),-125.6\left(\mathrm{ddd},{ }^{2} J(\mathrm{~F}-\mathrm{F})=304.7,{ }^{2} J(\mathrm{~F}-\mathrm{P})=102.8,{ }^{3} J(\mathrm{~F}-\right.$ $\left.\mathrm{H})=19.0, \mathrm{PCF}_{\mathrm{A}} \mathrm{F}_{\mathrm{B}} \mathrm{CHOH}\right) ;{ }^{1} \mathrm{H} \mathrm{NMR}\left(\mathrm{CDCl}_{3}\right): \delta 1.32\left(\mathrm{td}, 6 \mathrm{H},{ }^{3} \mathrm{~J}(\mathrm{H}-\mathrm{H})=7.1, \mathrm{C}_{3} \mathrm{CH}_{2} \mathrm{O}\right)$, $3.80\left(\mathrm{~s}, 3 \mathrm{H}, \mathrm{OC}_{3}\right), 4.0\left(\mathrm{~m}, 1 \mathrm{H}, \mathrm{PCF}_{2} \mathrm{CHOH}\right), 4.18\left(\mathrm{~m}, 4 \mathrm{H},{ }^{3} J(\mathrm{H}-\mathrm{H})=7.1, \mathrm{CH}_{3} \mathrm{C}_{2} \mathrm{O}\right), 5.06$ $\left(\mathrm{dm}, 1 \mathrm{H},{ }^{3} \mathrm{~J}=20.2, \mathrm{PCF}_{2} \mathrm{CHOH}\right), 6.90\left(\mathrm{~d}, 2 \mathrm{H},{ }^{3} \mathrm{~J}(\mathrm{H}-\mathrm{H})=8.6, \mathrm{H}_{\text {meta }}\right.$ of $\left.\mathrm{C}_{6} \mathrm{H}_{4} \mathrm{OCH}_{3}\right), 7.39(\mathrm{~d}$, $2 \mathrm{H},{ }^{3} J(\mathrm{H}-\mathrm{H})=8.6, \mathrm{H}_{\text {ortho }}$ of $\left.\mathrm{C}_{6} \mathrm{H}_{4} \mathrm{OCH}_{3}\right) ;{ }^{13} \mathrm{C} \mathrm{NMR}\left(\mathrm{CDCl}_{3}\right): \delta 16.5\left(\mathrm{~d},{ }^{3} J(\mathrm{C}-\mathrm{P})=4.6\right.$, $\left.\mathrm{CH}_{3} \mathrm{CH}_{2} \mathrm{O}\right), 55.5\left(\mathrm{~s}, \mathrm{OCH}_{3}\right), 65.2\left(\mathrm{dd},{ }^{2} \mathrm{~J}(\mathrm{C}-\mathrm{P})=7.2, J=12.0, \mathrm{CH}_{3} \underline{\mathrm{CH}}_{2} \mathrm{O}\right), 73.3(\mathrm{~m}, \mathrm{X}$ part of ABMX system, $\mathrm{PMCF}_{\mathrm{AFB}} \mathrm{CX} \mathrm{HOH}$ ), 113.8 (s, $\underline{\mathrm{C}}_{\text {meta }}$ of $\mathrm{C}_{6} \mathrm{H}_{4} \mathrm{OCH}_{3}$ ), 118.2 (ddd, $\mathrm{X}$ part of $\mathrm{ABMX}$ system, ${ }^{1} J(\mathrm{C}-\mathrm{F})=271.7,{ }^{1} J(\mathrm{C}-\mathrm{F})=265.8,{ }^{1} J(\mathrm{C}-\mathrm{P})=204.2, \mathrm{PMCX}_{\mathrm{CA}} \mathrm{FBCHOH}_{\mathrm{B}}$, $127.1\left(\mathrm{t},{ }^{3} J(\mathrm{C}-\mathrm{F})=6.0, \underline{\mathrm{C}}_{\mathrm{ipso}}\right.$ of $\left.\mathrm{C}_{6} \mathrm{H}_{4} \mathrm{OCH}_{3}\right), 129.6\left(\mathrm{~s}, \underline{\mathrm{C}}_{\text {ortho }}\right.$ of $\left.\mathrm{C}_{6} \mathrm{H}_{4} \mathrm{OCH}_{3}\right), 160.2\left(\mathrm{~s}, \underline{\mathrm{C}}_{\text {para }}\right.$ of $\left.\mathrm{C}_{6} \mathrm{H}_{4} \mathrm{OCH}_{3}\right)$.

$\mathrm{m} / \mathrm{z}(\mathrm{CI}+\mathrm{ve}) 324\left(\mathrm{M}^{+}, 5 \%\right)$. 
${ }^{31} \mathrm{P}$ NMR $\left(\mathrm{CDCl}_{3}\right): \delta+5.5\left(\mathrm{t},{ }^{2} J(\mathrm{P}-\mathrm{F})=104\right) ;{ }^{19} \mathrm{~F} \mathrm{NMR}\left(\mathrm{CDCl}_{3}\right): \delta-115.2\left(\mathrm{ddd},{ }^{2} J(\mathrm{~F}-\mathrm{F})=\right.$ $\left.304.7,{ }^{2} J(\mathrm{~F}-\mathrm{P})=99.0,{ }^{3} J(\mathrm{~F}-\mathrm{H})=7.6, \mathrm{PCF}_{\mathrm{A}} \mathrm{F}_{\mathrm{B}} \mathrm{CHOH}\right),-125.5\left(\mathrm{ddd},{ }^{2} J(\mathrm{~F}-\mathrm{F})=304.7,{ }^{2} J(\mathrm{~F}-\right.$ $\left.\mathrm{P})=106.6,{ }^{3} J(\mathrm{~F}-\mathrm{H})=19.0, \mathrm{PCF}_{\mathrm{A}} \mathrm{F}_{\mathrm{B}} \mathrm{CHOH}\right) ;{ }^{1} \mathrm{H} \mathrm{NMR}\left(\mathrm{CDCl}_{3}\right): \delta 1.33\left(\mathrm{td}, 6 \mathrm{H},{ }^{3} J(\mathrm{H}-\mathrm{H})=\right.$ 7.0, $\left.\mathrm{C}_{3} \mathrm{CH}_{2} \mathrm{O}\right), 2.95$ (s, $\left.6 \mathrm{H}, \mathrm{N}\left(\mathrm{C}_{3}\right)_{2}\right), 4.0\left(\mathrm{~m}, 1 \mathrm{H}, \mathrm{PCF}_{2} \mathrm{CHO} \underline{\mathrm{H}}\right), 4.24\left(\mathrm{~m}, 4 \mathrm{H}, \mathrm{CH}_{3} \underline{\mathrm{C}}_{2} \mathrm{O}\right)$, $5.03\left(\mathrm{dm}, 1 \mathrm{H},{ }^{3} J(\mathrm{H}-\mathrm{P})=20, \mathrm{PCF}_{2} \mathrm{CHOH}\right), 6.72\left(\mathrm{~d}, 2 \mathrm{H},{ }^{3} J(\mathrm{H}-\mathrm{H})=8.8, \mathrm{H}_{\text {meta }}\right.$ of $\left.\mathrm{C}_{6} \mathrm{H}_{4} \mathrm{~N}\left(\mathrm{CH}_{3}\right)_{2}\right), 7.33\left(\mathrm{~d}, 2 \mathrm{H},{ }^{3} J(\mathrm{H}-\mathrm{H})=8.3, \mathrm{H}_{\text {ortho }}\right.$ of $\left.\mathrm{C}_{6} \mathrm{H}_{4} \mathrm{~N}\left(\mathrm{CH}_{3}\right)_{2}\right) ;{ }^{13} \mathrm{C} \mathrm{NMR}\left(\mathrm{CDCl}_{3}\right): \delta$ $17.0\left(\mathrm{~d},{ }^{3} J(\mathrm{C}-\mathrm{P})=5.9, \underline{\mathrm{CH}}_{3} \mathrm{CH}_{2} \mathrm{O}\right), 41.1\left(\mathrm{~s}, \mathrm{~N}\left(\underline{\mathrm{CH}}_{3}\right)_{2}\right), 65.5\left(\mathrm{dd},{ }^{2} J(\mathrm{C}-\mathrm{P})=6.7, J=12.1\right.$, $\left.\mathrm{CH}_{3} \mathrm{CH}_{2} \mathrm{O}\right), 73.8\left(\mathrm{~m}, \mathrm{X}\right.$ part of $\mathrm{ABMX}$ system, ${ }^{2} J(\mathrm{C}-\mathrm{F})=26.6,{ }^{2} J(\mathrm{C}-\mathrm{F})=21.3,{ }^{2} J(\mathrm{C}-\mathrm{P})=$ 14.7, $\left.\mathrm{PMCF}_{\mathrm{A}} \mathrm{FBCX}_{\mathrm{B}} \mathrm{HOH}\right), 112.6$ (s, $\underline{\mathrm{C}}_{\text {meta }}$ of $\left.\mathrm{C}_{6} \mathrm{H}_{4} \mathrm{~N}\left(\underline{\mathrm{CH}}_{3}\right)_{2}\right), 118.8$ (m, X part of ABMX system, $\left.{ }^{1} J(\mathrm{C}-\mathrm{F})=270.1,{ }^{1} J(\mathrm{C}-\mathrm{F})=264.0,{ }^{1} J(\mathrm{C}-\mathrm{P})=204.5, \mathrm{PMCXFAFBCHOH}\right), 123.0(\mathrm{~d}$, ${ }^{3} J(\mathrm{C}-\mathrm{P})=4.9, \underline{\mathrm{C}}_{\text {ipso }}$ of $\left.\mathrm{C}_{6} \mathrm{H}_{4} \mathrm{~N}\left(\underline{\mathrm{CH}}_{3}\right)_{2}\right), 129.6\left(\mathrm{~s}, \underline{\mathrm{C}}_{\text {ortho }}\right.$ of $\left.\mathrm{C}_{6} \mathrm{H}_{4} \mathrm{~N}\left(\underline{\mathrm{CH}}_{3}\right)_{2}\right), 151.6\left(\mathrm{~s}, \underline{\mathrm{C}}_{\text {para }}\right.$ of $\left.\mathrm{C}_{6} \mathrm{H}_{4} \mathrm{~N}\left(\underline{\mathrm{CH}}_{3}\right)_{2}\right)$.

$\mathrm{m} / \mathrm{z}(\mathrm{EI}) 337\left(\mathrm{M}^{+}, 7 \%\right), 150$ (100).

\subsubsection{Diethyl 1,1-difluoro 2-(2'-furyl) 2-hydroxyethylphosphonate 7i}

${ }^{31} \mathrm{P}$ NMR $\left(\mathrm{CDCl}_{3}\right): \delta+4.3\left(\mathrm{t},{ }^{2} J(\mathrm{P}-\mathrm{F})=101\right) ;{ }^{19} \mathrm{~F} \mathrm{NMR}\left(\mathrm{CDCl}_{3}\right): \delta-115.9\left(\mathrm{ddd},{ }^{2} J(\mathrm{~F}-\mathrm{F})=\right.$ $\left.304.7,{ }^{2} J(\mathrm{~F}-\mathrm{P})=99.0,{ }^{3} J(\mathrm{~F}-\mathrm{H})=7.6, \mathrm{PCF}_{\mathrm{A}} \mathrm{F}_{\mathrm{B}} \mathrm{CHOH}\right),-123.6\left(\mathrm{ddd},{ }^{2} J(\mathrm{~F}-\mathrm{F})=304.7,{ }^{2} J(\mathrm{~F}-\right.$ $\left.\mathrm{P})=102.8,{ }^{3} J(\mathrm{~F}-\mathrm{H})=19.0, \mathrm{PCF}_{\mathrm{A}} \underline{\mathrm{F}}_{\mathrm{B}} \mathrm{CHOH}\right) ;{ }^{1} \mathrm{H} \mathrm{NMR}\left(\mathrm{CDCl}_{3}\right): \delta 1.34\left(\mathrm{td}, 6 \mathrm{H},{ }^{3} J(\mathrm{H}-\mathrm{H})=\right.$ $\left.7.0,{ }^{4} J(\mathrm{H}-\mathrm{P})=4.5, \mathrm{CH}_{3} \mathrm{CH}_{2} \mathrm{O}\right), 4.09\left(\mathrm{~m}, 1 \mathrm{H}, \mathrm{PCF}_{2} \mathrm{CHOH}\right), 4.25\left(\mathrm{qd}, 4 \mathrm{H},{ }^{3} J(\mathrm{H}-\mathrm{P})=8.9\right.$, $\left.{ }^{3} J(\mathrm{H}-\mathrm{H})=7.0, \mathrm{CH}_{3} \underline{\mathrm{C}}_{2} \mathrm{O}\right), 5.15\left(\mathrm{dm}, 1 \mathrm{H}, \mathrm{PCF}_{2} \mathrm{C} \underline{\mathrm{HOH}}\right), 6.40\left(\mathrm{dd}, 1 \mathrm{H},{ }^{3} J(\mathrm{H}-\mathrm{H})=3.3,{ }^{3} J(\mathrm{H}-\right.$ $\mathrm{H})=1.8, \underline{\mathrm{H}}_{4}$ of $\left.\mathrm{C}_{4} \mathrm{H}_{3} \mathrm{O}\right), 6.52\left(\mathrm{~d}_{\text {large }}, 1 \mathrm{H},{ }^{3} J(\mathrm{H}-\mathrm{H})=3.3, \underline{\mathrm{H}}_{3}\right.$ of $\left.\mathrm{C}_{4} \mathrm{H}_{3} \mathrm{O}\right), 7.45\left(\mathrm{~m}, 1 \mathrm{H}, \underline{\mathrm{H}}_{5}\right.$ of

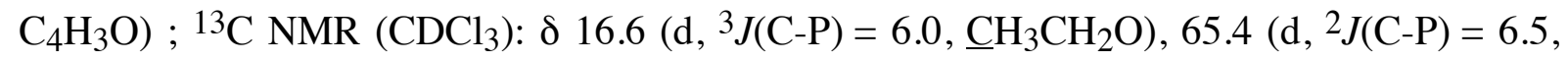
$\mathrm{CH}_{3} \mathrm{CH}_{2} \mathrm{O}$ ), 68.1 (ddd, $\mathrm{X}$ part of ABMX system, ${ }^{2} J(\mathrm{C}-\mathrm{F})=27.1,{ }^{2} J(\mathrm{C}-\mathrm{F})=21.9,{ }^{2} J(\mathrm{C}-\mathrm{P})=$ 16.6, $\mathrm{PMCF}_{\mathrm{MFB}} \mathrm{CX} \mathrm{HOH}$ ), 110.3 (s, $\underline{\mathrm{C}}_{3}$ of $\mathrm{C}_{4} \mathrm{H}_{3} \mathrm{O}$ ), 110.9 (s, $\underline{\mathrm{C}}_{4}$ of $\mathrm{C}_{4} \mathrm{H}_{3} \mathrm{O}$ ), 118.3 (ddd, $\mathrm{X}$ part of $\mathrm{ABMX}$ system, ${ }^{1} J(\mathrm{C}-\mathrm{F})=271.7, \quad{ }^{1} J(\mathrm{C}-\mathrm{F})=265.0, \quad{ }^{1} J(\mathrm{C}-\mathrm{P})=209.8$, PMCXFAFBCHOH), 143.3 (s, $\underline{\mathrm{C}}_{5}$ of $\left.\mathrm{C}_{4} \mathrm{H}_{3} \mathrm{O}\right), 149.6\left(\mathrm{dd},{ }^{3} J(\mathrm{C}-\mathrm{P})=6.2,{ }^{3} J(\mathrm{C}-\mathrm{P})=2.7, \underline{\mathrm{C}}_{2}\right.$ of $\left.\mathrm{C}_{4} \mathrm{H}_{3} \mathrm{O}\right)$.

$\mathrm{m} / \mathrm{z}(\mathrm{EI}) 284\left(\mathrm{M}^{+}, 10 \%\right), 188(73), 161(75), 132(100)$.

\subsubsection{Diethyl 1,1-difluoro 2-(2'-thienyl) 2-hydroxyethylphosphonate $7 \boldsymbol{j}$}

${ }^{31} \mathrm{P}$ NMR $\left(\mathrm{CDCl}_{3}\right): \delta+4.8\left(\mathrm{t},{ }^{2} J(\mathrm{P}-\mathrm{F})=101\right) ;{ }^{19} \mathrm{~F} \mathrm{NMR}\left(\mathrm{CDCl}_{3}\right): \delta-115.0\left(\mathrm{ddd},{ }^{2} J(\mathrm{~F}-\mathrm{F})=\right.$ $\left.299.0,{ }^{2} J(\mathrm{~F}-\mathrm{P})=99.0,{ }^{3} J(\mathrm{~F}-\mathrm{H})=7.6, \mathrm{PCF}_{\mathrm{A}} \mathrm{F}_{\mathrm{B}} \mathrm{CHOH}\right),-125.2\left(\mathrm{ddd},{ }^{2} J(\mathrm{~F}-\mathrm{F})=299.0,{ }^{2} J(\mathrm{~F}-\right.$ $\left.\mathrm{P})=102.8,{ }^{3} J(\mathrm{~F}-\mathrm{H})=19.0, \mathrm{PCF}_{\mathrm{A}} \mathrm{F}_{\mathrm{B}} \mathrm{CHOH}\right) ;{ }^{1} \mathrm{H} \mathrm{NMR}\left(\mathrm{CDCl}_{3}\right): \delta 1.34\left(\mathrm{q}, 6 \mathrm{H},{ }^{3} J(\mathrm{H}-\mathrm{H})=\right.$ $\left.{ }^{4} J(\mathrm{H}-\mathrm{P})=7.0, \mathrm{C}_{3} \mathrm{CH}_{2} \mathrm{O}\right), 1.8\left(\mathrm{~m}, 1 \mathrm{H}, \mathrm{PCF}_{2} \mathrm{CHO} \underline{\mathrm{H}}\right), 4.25\left(\mathrm{p}, 4 \mathrm{H},{ }^{3} J(\mathrm{H}-\mathrm{P})={ }^{3} J(\mathrm{H}-\mathrm{H})=7.0\right.$, $\left.\mathrm{CH}_{3} \mathrm{CH}_{2} \mathrm{O}\right), 5.39\left(\mathrm{dm}, 1 \mathrm{H},{ }^{3} J(\mathrm{H}-\mathrm{P})=19.5, \mathrm{PCF}_{2} \mathrm{CHOH}\right), 7.03\left(\mathrm{dd}, 1 \mathrm{H},{ }^{3} J(\mathrm{H}-\mathrm{H})=5.0,{ }^{3} J(\mathrm{H}-\right.$ $\mathrm{H})=3.6, \underline{\mathrm{H}}_{4}$ of $\left.\mathrm{C}_{4} \mathrm{H}_{3} \mathrm{~S}\right), 7.18\left(\mathrm{~d}, 1 \mathrm{H},{ }^{3} J(\mathrm{H}-\mathrm{H})=3.6, \underline{\mathrm{H}}_{3}\right.$ of $\left.\mathrm{C}_{4} \mathrm{H}_{3} \mathrm{~S}\right), 7.36\left(\mathrm{dd}, 1 \mathrm{H},{ }^{3} J(\mathrm{H}-\mathrm{H})=\right.$ $5.0,{ }^{4} J(\mathrm{H}-\mathrm{H})=1.2, \underline{\mathrm{H}}_{5}$ of $\left.\mathrm{C}_{4} \mathrm{H}_{3} \mathrm{~S}\right) ;{ }^{13} \mathrm{C} \mathrm{NMR}\left(\mathrm{CDCl}_{3}\right): \delta 16.9\left(\mathrm{~d},{ }^{3} J(\mathrm{C}-\mathrm{P})=5.6, \underline{\mathrm{CH}}_{3} \mathrm{CH}_{2} \mathrm{O}\right)$, 
$65.7\left(\mathrm{~d},{ }^{2} J(\mathrm{C}-\mathrm{P})=7.0, \mathrm{CH}_{3} \underline{\mathrm{CH}}_{2} \mathrm{O}\right), 70.7\left(\mathrm{ddd}, \mathrm{X}\right.$ part of $\mathrm{ABMX}$ system, ${ }^{2} J(\mathrm{C}-\mathrm{F})=27.5,{ }^{2} J(\mathrm{C}-$ $\left.\mathrm{F})=22.4,{ }^{2} J(\mathrm{C}-\mathrm{P})=16.2, \mathrm{PMCFAFB} C X \mathrm{HOH}\right), 118.1\left(\mathrm{ddd}, \mathrm{X}\right.$ part of ABMX system, ${ }^{1} \mathrm{~J}(\mathrm{C}-$ $\left.\mathrm{F})=272.6,{ }^{1} J(\mathrm{C}-\mathrm{F})=264.8,{ }^{1} J(\mathrm{C}-\mathrm{P})=206.6, \mathrm{PMCXF}_{\mathrm{AFBCHOH}}\right), 127.1\left(\mathrm{~s}, \underline{\mathrm{C}}_{3}\right.$ of $\left.\mathrm{C}_{4} \mathrm{H}_{3} \mathrm{~S}\right)$, $127.2\left(\mathrm{~s}, \underline{\mathrm{C}}_{4}\right.$ of $\left.\mathrm{C}_{4} \mathrm{H}_{3} \mathrm{~S}\right), 127.7\left(\mathrm{~s}, \underline{\mathrm{C}}_{5}\right.$ of $\left.\mathrm{C}_{4} \mathrm{H}_{3} \mathrm{~S}\right), 138.4\left(\mathrm{~d},{ }^{3} \mathrm{~J}(\mathrm{C}-\mathrm{P})=6.7, \underline{\mathrm{C}}_{2}\right.$ of $\left.\mathrm{C}_{4} \mathrm{H}_{3} \mathrm{~S}\right)$.

m/z (EI) $300\left(\mathrm{M}^{+}, 13 \%\right), 188$ (80), 161 (93), 132 (100).

\subsubsection{Diethyl 1,1-difluoro 2-(2'-pyridyl) 2-hydroxyethylphosphonate $7 \boldsymbol{k}$}

${ }^{31} \mathrm{P}$ NMR $\left(\mathrm{CDCl}_{3}\right): \delta+4.3\left(\mathrm{t},{ }^{2} J(\mathrm{P}-\mathrm{F})=101\right) ;{ }^{19} \mathrm{~F} \mathrm{NMR}\left(\mathrm{CDCl}_{3}\right): \delta-114.6\left(\mathrm{dd},{ }^{2} J(\mathrm{~F}-\mathrm{F})=\right.$ $\left.304.7,{ }^{2} J(\mathrm{~F}-\mathrm{P})=99.0, \mathrm{PCF}_{\mathrm{A}} \mathrm{F}_{\mathrm{B}} \mathrm{CHOH}\right),-125.6\left(\mathrm{ddd},{ }^{2} J(\mathrm{~F}-\mathrm{F})=304.7,{ }^{2} J(\mathrm{~F}-\mathrm{P})=102.8,{ }^{3} J(\mathrm{~F}-\right.$ $\left.\mathrm{H})=19.0, \mathrm{PCF}_{\mathrm{A}} \underline{F}_{\mathrm{B}} \mathrm{CHOH}\right) ;{ }^{1} \mathrm{H} \mathrm{NMR}\left(\mathrm{CDCl}_{3}\right): \delta 1.35\left(\mathrm{td}, 6 \mathrm{H},{ }^{3} J(\mathrm{H}-\mathrm{H})=7.1,{ }^{4} J(\mathrm{H}-\mathrm{P})=2.7\right.$, $\left.\mathrm{CH}_{3} \mathrm{CH}_{2} \mathrm{O}\right), 4.26\left(\mathrm{qd}, 4 \mathrm{H},{ }^{3} J(\mathrm{H}-\mathrm{P})=8.0,{ }^{3} J(\mathrm{H}-\mathrm{H})=7.1, \mathrm{CH}_{3} \mathrm{CH}_{2} \mathrm{O}\right), 5.20\left(\mathrm{dt}, 1 \mathrm{H},{ }^{3} J(\mathrm{H}-\mathrm{P})=\right.$ $\left.20.8,{ }^{3} J(\mathrm{H}-\mathrm{F})=4.5, \mathrm{PCF}_{2} \mathrm{C} \underline{\mathrm{HOH}}\right), 5.67\left(\mathrm{~s}_{\text {large }}, 1 \mathrm{H}, \mathrm{PCF}_{2} \mathrm{CHO} \underline{\mathrm{H}}\right), 7.35\left(\mathrm{dd}, 1 \mathrm{H},{ }^{3} J(\mathrm{H}-\mathrm{H})=7.7\right.$ et $4.8, \underline{\mathrm{H}}_{5}$ of $\left.\mathrm{C}_{5} \mathrm{H}_{4} \mathrm{~N}\right), 7.48\left(\mathrm{~d}_{\text {large }}, 1 \mathrm{H},{ }^{3} J(\mathrm{H}-\mathrm{H})=7.7, \underline{\mathrm{H}}_{3}\right.$ of $\left.\mathrm{C}_{5} \mathrm{H}_{4} \mathrm{~N}\right), 7.78\left(\mathrm{tt}, 1 \mathrm{H},{ }^{3} J(\mathrm{H}-\mathrm{H})=\right.$ $7.7,{ }^{4} J(\mathrm{H}-\mathrm{H})={ }^{6} J(\mathrm{H}-\mathrm{P})=1.2, \underline{\mathrm{H}}_{4}$ of $\left.\mathrm{C}_{5} \mathrm{H}_{4} \mathrm{~N}\right), 8.60\left(\mathrm{dd}, 1 \mathrm{H},{ }^{3} J(\mathrm{H}-\mathrm{H})=4.8,{ }^{4} J(\mathrm{H}-\mathrm{H})=1.2, \underline{\mathrm{H}}_{6}\right.$ of $\left.\mathrm{C}_{5} \mathrm{H}_{4} \mathrm{~N}\right) ;{ }^{13} \mathrm{C} \mathrm{NMR}\left(\mathrm{CDCl}_{3}\right): \delta 15.9\left(\mathrm{~d},{ }^{3} J(\mathrm{C}-\mathrm{P})=5.4, \mathrm{CH}_{3} \mathrm{CH}_{2} \mathrm{O}\right), 64.3\left(\mathrm{~d},{ }^{2} J(\mathrm{C}-\mathrm{P})=6.3\right.$, $\mathrm{CH}_{3} \underline{\mathrm{CH}}_{2} \mathrm{O}$ ), 72.5 (ddd, $\mathrm{X}$ part of ABMX system, ${ }^{2} J(\mathrm{C}-\mathrm{F})=26.1,{ }^{2} J(\mathrm{C}-\mathrm{F})=21.9,{ }^{2} J(\mathrm{C}-\mathrm{P})=$ 13.6, $\left.\mathrm{PMCF}_{\mathrm{A}} \mathrm{FBCX}_{\mathrm{X}} \mathrm{HOH}\right), 118.4$ (ddd, $\mathrm{X}$ part of $\mathrm{ABMX}$ system, ${ }^{1} J(\mathrm{C}-\mathrm{F})=272.6,{ }^{1} \mathrm{~J}(\mathrm{C}-\mathrm{F})=$ 264.8, $\left.{ }^{1} J(\mathrm{C}-\mathrm{P})=209.2, \mathrm{PMCXFAFBCHOH}_{\mathrm{M}}\right), 123.0\left(\mathrm{~s}, \underline{\mathrm{C}}_{3}\right.$ of $\left.\mathrm{C}_{5} \mathrm{H}_{4} \mathrm{~N}\right), 123.5$ (s, $\underline{\mathrm{C}}_{5}$ of $\left.\mathrm{C}_{5} \mathrm{H}_{4} \mathrm{~N}\right), 136.5\left(\mathrm{~s}, \underline{\mathrm{C}}_{4}\right.$ of $\left.\mathrm{C}_{5} \mathrm{H}_{4} \mathrm{~N}\right), 147.9\left(\mathrm{~s}, \underline{\mathrm{C}}_{6}\right.$ of $\left.\mathrm{C}_{5} \mathrm{H}_{4} \mathrm{~N}\right), 153.9\left(\mathrm{~d},{ }^{3} J(\mathrm{C}-\mathrm{P})=6.1, \underline{\mathrm{C}}_{2}\right.$ of $\left.\mathrm{C}_{5} \mathrm{H}_{4} \mathrm{~N}\right)$.

$\mathrm{m} / \mathrm{z}(\mathrm{EI}) 296\left(\mathrm{M}+\mathrm{H}^{+}, 5 \%\right), 161(8), 132(17), 108$ (100).

\subsection{General procedure for the condensation of 2 with ketones}

A $250 \mathrm{~mL}$ reactor equipped as above was charged with anhydrous $\mathrm{LiBr}(1.31 \mathrm{~g}, 0.015 \mathrm{~mol}$, dried by heating at $150^{\circ} \mathrm{C}$ under $0.1 \mathrm{~mm} \mathrm{Hg}$ vacuum over one hour) and THF (15 mL) and heated at $50^{\circ} \mathrm{C}$ until complete dissolution. $\mathrm{PrMgCl}\left(7.5 \mathrm{~mL}\right.$ of $1.90 \mathrm{M} \mathrm{Et}_{2} \mathrm{O}$ solution, 0.015 $\mathrm{mol})$ and THF $(10 \mathrm{~mL})$ were added into the reactor at $-20^{\circ} \mathrm{C}$. The solution was cooled to $78^{\circ} \mathrm{C}$ and a solution of $1(4.00 \mathrm{~g}, 0.015 \mathrm{~mol})$ in THF $(20 \mathrm{~mL})$ was added dropwise. The resulting mixture was stirred for $5 \mathrm{~min}$ at $-78^{\circ} \mathrm{C}$ then allow ta warm up to $-40^{\circ} \mathrm{C}$. At this temperature a solution of ketone $(0.01 \mathrm{~mol})$ in $\mathrm{THF}(20 \mathrm{~mL})$ was added dropwise. The resulting mixture was stirred for $15 \mathrm{~min}$ at $-40^{\circ} \mathrm{C}$ then was allowed to warm up to $0^{\circ} \mathrm{C}$ within one hour and from $0^{\circ} \mathrm{C}$ to room temperature for an additional hour. The reaction mixture was poured into an ice-cold mixture of $\mathrm{HCl}\left(20 \mathrm{~mL}\right.$ of $2 \mathrm{M}$ solution) and $\mathrm{CH}_{2} \mathrm{Cl}_{2}(20 \mathrm{~mL})$. The aqueous layer was extracted with $\mathrm{CH}_{2} \mathrm{Cl}_{2}(2 \times 20 \mathrm{~mL})$. The extracts were dried $\left(\mathrm{MgSO}_{4}\right)$ and the solvents were removed under reduced pressure to give the crude product 8 and the excess of 3 which was prior eliminated by heating the crude mixture at $70^{\circ} \mathrm{C}$ under $0.5 \mathrm{~mm} \mathrm{Hg}$ for 
one hour. Then $\mathbf{8}$ was purified either by bulb-to-bulb distillation or by column chromatography (see Table 3 ).

\subsubsection{Diethyl 1,1-difluoro 1-(1'-hydroxy)cyclohexyl methylphosphonate $8 \mathbf{a}$}

${ }^{31} \mathrm{P}$ NMR $\left(\mathrm{CDCl}_{3}\right): \delta+5.5\left(\mathrm{t},{ }^{2} J(\mathrm{P}-\mathrm{F})=107\right) ;{ }^{19} \mathrm{~F} \mathrm{NMR}\left(\mathrm{CDCl}_{3}\right): \delta-121.5\left(\mathrm{~d},{ }^{2} J(\mathrm{~F}-\mathrm{P})=\right.$ 106.6, $\left.\mathrm{PCF}_{2} \mathrm{COH}\right) ;{ }^{1} \mathrm{H} \mathrm{NMR}\left(\mathrm{CDCl}_{3}\right): \delta 1.2\left(\mathrm{~m}, 1 \mathrm{H}, \mathrm{COH}\left(\mathrm{CH}_{2} \mathrm{CH}_{2}\right)_{2} \mathrm{CH}_{\mathrm{ax}} \mathrm{H}_{\mathrm{eq}}\right), 1.37(\mathrm{t}, 6 \mathrm{H}$, $\left.{ }^{3} J(\mathrm{H}-\mathrm{H})=7.1, \mathrm{CH}_{3} \mathrm{CH}_{2} \mathrm{O}\right), 1.61\left(\mathrm{~m}, 7 \mathrm{H}, \mathrm{COH}\left(\mathrm{CH}_{\mathrm{ax}} \mathrm{H}_{\mathrm{eq}} \underline{\mathrm{H}}_{2}\right)_{2} \mathrm{CH}_{\mathrm{ax}} \underline{\mathrm{H}}_{\mathrm{eq}}\right), 1.87\left(\mathrm{~d}_{\text {large }}, 2 \mathrm{H}\right.$, $\left.{ }^{2} J(\mathrm{H}-\mathrm{H})=11.9, \mathrm{COH}\left(\mathrm{CH}_{\mathrm{ax}} \underline{\mathrm{H}}_{\mathrm{eq}} \mathrm{CH}_{2}\right)_{2} \mathrm{CH}_{2}\right), 2.99\left(\mathrm{~s}, 1 \mathrm{H}, \mathrm{PCF}_{2} \mathrm{CO} \underline{\mathrm{H}}\right), 4.28\left(\mathrm{p}, 4 \mathrm{H},{ }^{3} J(\mathrm{H}-\mathrm{P})=\right.$ $\left.{ }^{3} J(\mathrm{H}-\mathrm{H})=7.1, \mathrm{CH}_{3} \mathrm{CH}_{2} \mathrm{O}\right) ;{ }^{13} \mathrm{C} \mathrm{NMR}\left(\mathrm{CDCl}_{3}\right): \delta 16.9\left(\mathrm{~d},{ }^{3} J(\mathrm{C}-\mathrm{P})=5.9, \mathrm{CH}_{3} \mathrm{CH}_{2} \mathrm{O}\right), 21.1$ (s, $\left.\mathrm{COH}\left(\mathrm{CH}_{2} \underline{\mathrm{CH}}_{2}\right)_{2} \mathrm{CH}_{2}\right), \quad 26.0 \quad$ (s, $\left.\quad \mathrm{COH}\left(\mathrm{CH}_{2} \mathrm{CH}_{2}\right)_{2} \mathrm{CH}_{2}\right), \quad 30.3 \quad(\mathrm{~d}, \quad 3 J(\mathrm{C}-\mathrm{P})=2.7$, $\left.\mathrm{COH}\left(\mathrm{CH}_{2} \mathrm{CH}_{2}\right)_{2} \mathrm{CH}_{2}\right), 65.5\left(\mathrm{~d},{ }^{2} J(\mathrm{C}-\mathrm{P})=6.8, \mathrm{CH}_{3} \mathrm{CH}_{2} \mathrm{O}\right), 74.5\left(\mathrm{td},{ }^{2} J(\mathrm{C}-\mathrm{F})=20.9,{ }^{2} J(\mathrm{C}-\mathrm{P})=\right.$ 13.4, $\left.\mathrm{PCF}_{2} \underline{\mathrm{COH}}\right), 120.9\left(\mathrm{td},{ }^{1} J(\mathrm{C}-\mathrm{F})=271.0,{ }^{1} J(\mathrm{C}-\mathrm{P})=200.3, \mathrm{PCF}_{2} \mathrm{COH}\right)$.

$\mathrm{m} / \mathrm{z}(\mathrm{EI}) 287\left(\mathrm{M}+\mathrm{H}^{+}, 6 \%\right), 188$ (100), $161(90), 132(79)$.

\subsubsection{Diethyl 1,1-difluoro 1-(1'-hydroxy-4'-tertiobutyl)cyclohexyl methylphosphonate $8 \boldsymbol{b}$}

${ }^{31} \mathrm{P} \mathrm{NMR}\left(\mathrm{CDCl}_{3}\right): \delta+5.6\left(\mathrm{t},{ }^{2} J(\mathrm{P}-\mathrm{F})=106\right) ;{ }^{19} \mathrm{~F} \mathrm{NMR}\left(\mathrm{CDCl}_{3}\right): \delta-121.5\left(\mathrm{~d},{ }^{2} J(\mathrm{~F}-\mathrm{P})=\right.$ 106.6, $\left.\mathrm{PCF}_{2} \mathrm{COH}\right) ;{ }^{1} \mathrm{H} \mathrm{NMR}\left(\mathrm{CDCl}_{3}\right): \delta 0.93\left(\mathrm{~s}, 9 \mathrm{H}, \mathrm{C}\left(\mathrm{CH}_{3}\right)_{3}\right), 1.05\left(\mathrm{t}_{\text {large }}, 1 \mathrm{H},{ }^{3} J(\mathrm{Hax}-\right.$ $\left.\mathrm{Hax})=11.4, \mathrm{COH}\left(\mathrm{CH}_{2} \mathrm{CH}_{2}\right)_{2} \mathrm{C}_{\mathrm{ax}}\right), 1.45\left(\mathrm{t}, 6 \mathrm{H},{ }^{3} \mathrm{~J}(\mathrm{H}-\mathrm{H})=7.1, \mathrm{C}_{3} \mathrm{CH}_{2} \mathrm{O}\right), 1.52-1.75(\mathrm{~m}$, $\left.6 \mathrm{H}, \quad \mathrm{COH}\left(\mathrm{CH}_{\mathrm{ax}} \mathrm{H}_{\mathrm{eq}} \mathrm{CH}_{2}\right)_{2} \mathrm{CH}_{\mathrm{ax}}\right), \quad 2.01 \quad\left(\mathrm{~d}_{\text {large }}, \quad 2 \mathrm{H}, \quad 2 J(\mathrm{H}-\mathrm{H})=\quad 12.4\right.$, $\left.\mathrm{COH}\left(\mathrm{CH}_{\mathrm{ax}} \underline{\mathrm{H}}_{\mathrm{eq}} \mathrm{CH}_{2}\right)_{2} \mathrm{CH}_{\mathrm{ax}}\right), 2.85\left(\right.$ slarge $\left., 1 \mathrm{H}, \mathrm{PCF}_{2} \mathrm{CO} \underline{\mathrm{H}}\right), 4.36\left(\mathrm{p}, 4 \mathrm{H},{ }^{3} J(\mathrm{H}-\mathrm{P})={ }^{3} J(\mathrm{H}-\mathrm{H})=\right.$ 7.1, $\left.\mathrm{CH}_{3} \mathrm{CH}_{2} \mathrm{O}\right) ;{ }^{13} \mathrm{C} \mathrm{NMR}\left(\mathrm{CDCl}_{3}\right): \delta 17.0\left(\mathrm{~d},{ }^{3} J(\mathrm{C}-\mathrm{P})=5.9, \underline{\mathrm{CH}}_{3} \mathrm{CH}_{2} \mathrm{O}\right), 22.0(\mathrm{~s}$, $\left.\mathrm{COH}\left(\mathrm{CH}_{2} \underline{\mathrm{CH}_{2}}\right)_{2} \mathrm{CH}\right), 28.1\left(\mathrm{~s}, \mathrm{C}\left(\underline{\mathrm{CH}}_{3}\right)_{3}\right), 30.8$ (d, $\left.{ }^{3} \mathrm{~J}(\mathrm{C}-\mathrm{P})=2.3, \mathrm{COH}\left(\underline{\mathrm{CH}}_{2} \mathrm{CH}_{2}\right)_{2} \mathrm{CH}\right), 33.3$ $\left(\mathrm{s}, \underline{\mathrm{C}}\left(\mathrm{CH}_{3}\right)_{3}\right), 48.0\left(\mathrm{~s}, \underline{\mathrm{CHC}}\left(\mathrm{CH}_{3}\right)_{3}\right), 65.5\left(\mathrm{~d},{ }^{2} J(\mathrm{C}-\mathrm{P})=6.9, \mathrm{CH}_{3} \underline{\mathrm{CH}}_{2} \mathrm{O}\right), 74.3\left(\mathrm{td},{ }^{2} J(\mathrm{C}-\mathrm{F})=\right.$ $\left.20.8,{ }^{2} J(\mathrm{C}-\mathrm{P})=13.3, \mathrm{PCF}_{2} \underline{\mathrm{COH}}\right), 121.0\left(\mathrm{td},{ }^{1} J(\mathrm{C}-\mathrm{F})=270.9,{ }^{1} J(\mathrm{C}-\mathrm{P})=200.4, \mathrm{PCF}_{2} \mathrm{COH}\right)$.

$\mathrm{m} / \mathrm{z}(\mathrm{CI}+\mathrm{ve}) 343\left(\mathrm{M}+\mathrm{H}^{+}, 100 \%\right)$.

\subsubsection{Diethyl 1,1-difluoro 1-(1'-hydroxy-1',2',3',4'-tetrahydro)naphtyl methylphosphonate 8 c}

${ }^{31} \mathrm{P} \mathrm{NMR}\left(\mathrm{CDCl}_{3}\right): \delta+5.4\left(\mathrm{dd},{ }^{2} J(\mathrm{P}-\mathrm{F})=133\right.$ and 99$) ;{ }^{1} \mathrm{H} \mathrm{NMR}\left(\mathrm{CDCl}_{3}\right): \delta 1.31(\mathrm{t}, 3 \mathrm{H}$, $\left.{ }^{3} J(\mathrm{H}-\mathrm{H})=7.1, \mathrm{CH}_{3} \mathrm{CH}_{2} \mathrm{O}_{\mathrm{A}}\right), 1.36\left(\mathrm{t}, 3 \mathrm{H},{ }^{3} J(\mathrm{H}-\mathrm{H})=7.1, \mathrm{CH}_{3} \mathrm{CH}_{2} \mathrm{O}_{\mathrm{B}}\right), 1.8-2.0\left(\mathrm{~m}, 3 \mathrm{H}, \mathrm{H}_{3^{\prime}}\right.$ and $\mathrm{H}_{2^{\prime}}$ pseudo eq $), 2.5\left(\mathrm{~m}, 1 \mathrm{H}, \mathrm{H}_{2^{\prime}}\right.$ pseudo ax $), 2.79\left(\mathrm{t}, 2 \mathrm{H},{ }^{3} J(\mathrm{H}-\mathrm{H})=6.0, \underline{\mathrm{H}}_{4}{ }^{\prime}\right), 3.29(\mathrm{~s}, 1 \mathrm{H}$, $\left.\mathrm{PCF}_{2} \mathrm{CO} \underline{\mathrm{H}}\right), 4.2\left(\mathrm{~m}, 2 \mathrm{H}, \mathrm{CH}_{3} \underline{\mathrm{H}}_{2} \mathrm{O}_{\mathrm{A}}\right), 4.26\left(\mathrm{p}, 2 \mathrm{H},{ }^{3} J(\mathrm{H}-\mathrm{P})={ }^{3} J(\mathrm{H}-\mathrm{H})=7.1, \mathrm{CH}_{3} \mathrm{CH}_{2} \mathrm{O}_{\mathrm{B}}\right)$, $7.10\left(\mathrm{~m}, 1 \mathrm{H}, \underline{\mathrm{H}}_{6^{\prime}}\right), 7.23\left(\mathrm{~m}, 2 \mathrm{H}, \underline{\mathrm{H}}_{5^{\prime}}\right.$ and $\left.7^{\prime}\right), 7.75\left(\mathrm{~m}, 1 \mathrm{H}, \underline{\mathrm{H}}_{8^{\prime}}\right) . ;{ }^{13} \mathrm{C} \mathrm{NMR}\left(\mathrm{CDCl}_{3}\right): \delta 16.4(\mathrm{~d}$, $\left.{ }^{3} J(\mathrm{C}-\mathrm{P})=5.9, \underline{\mathrm{CH}}_{3} \mathrm{CH}_{2} \mathrm{O}\right), 18.8\left(\mathrm{~d},{ }^{4} J(\mathrm{C}-\mathrm{P})=3.4, \underline{\mathrm{C}}_{3}{ }^{\prime}\right), 29.4\left(\mathrm{~s}, \underline{\mathrm{C}}_{4}{ }^{\prime}\right), 33.8\left(\mathrm{~d},{ }^{3} J(\mathrm{C}-\mathrm{P})=3.7\right.$, $\left.\underline{\mathrm{C}}_{2^{\prime}}\right), 64.6\left(\mathrm{~d},{ }^{2} J(\mathrm{C}-\mathrm{P})=5.9, \mathrm{CH}_{3} \underline{\mathrm{CH}}_{2} \mathrm{O}\right), 73.7\left(\mathrm{q},{ }^{2} J(\mathrm{C}-\mathrm{F})=20.7,{ }^{2} J(\mathrm{C}-\mathrm{P})=17.5, \mathrm{PCF}_{2} \underline{\mathrm{COH}}\right)$, $111.6\left(\mathrm{q},{ }^{1} J(\mathrm{C}-\mathrm{F})=257.8,{ }^{1} J(\mathrm{C}-\mathrm{P})=212.3, \mathrm{PCF}_{2} \mathrm{COH}\right), 125.7\left(\mathrm{~s}, \underline{\mathrm{C}}_{6}{ }^{\prime}\right), 128.3\left(\mathrm{~s}, \underline{\mathrm{C}}_{7^{\prime}}\right), 128.7$ $\left(\mathrm{s}, \underline{\mathrm{C}}_{5^{\prime}}\right), 129.4\left(\mathrm{~d},{ }^{4} J(\mathrm{C}-\mathrm{P})=2.9, \underline{\mathrm{C}}_{10^{\prime}}\right), 134.2\left(\mathrm{~d},{ }^{3} J(\mathrm{C}-\mathrm{P})=5.2, \underline{\mathrm{C}}_{9^{\prime}}\right), 139.2\left(\mathrm{~s}, \underline{\mathrm{C}}_{8^{\prime}}\right)$.

$\mathrm{m} / \mathrm{z}(\mathrm{EI}) 334\left(\mathrm{M}^{+}, 2 \%\right), 161$ (29), 147 (100). 
4.7.4. Diethyl 1,1-difluoro 2-hydroxy 2-methyl 2-(2'-thienyl) ethylphosphonate $8 d$

${ }^{31} \mathrm{P}$ NMR $\left(\mathrm{CDCl}_{3}\right): \delta+5.2\left(\mathrm{t},{ }^{2} J(\mathrm{P}-\mathrm{F})=103\right) ;{ }^{1} \mathrm{H} \mathrm{NMR}\left(\mathrm{CDCl}_{3}\right): \delta 1.14\left(\mathrm{t}, 3 \mathrm{H},{ }^{3} J(\mathrm{H}-\mathrm{H})=\right.$ 7.1, $\left.\mathrm{CH}_{3} \mathrm{CH}_{2} \mathrm{O}_{\mathrm{A}}\right), 1.37\left(\mathrm{t}, 3 \mathrm{H},{ }^{3} J(\mathrm{H}-\mathrm{H})=7.1, \mathrm{CH}_{3} \mathrm{CH}_{2} \mathrm{O}_{\mathrm{B}}\right), 1.73\left(\mathrm{t}, 3 \mathrm{H}, \mathrm{CH}_{3}\right), 3.7-4.2(\mathrm{~m}$, $\left.2 \mathrm{H}, \mathrm{CH}_{3} \mathrm{CH}_{2} \mathrm{O}_{\mathrm{A}}\right), 4.29\left(\mathrm{p}, 2 \mathrm{H},{ }^{3} J(\mathrm{H}-\mathrm{P})={ }^{3} J(\mathrm{H}-\mathrm{H})=7.1, \mathrm{CH}_{3} \mathrm{CH}_{2} \mathrm{O}_{\mathrm{B}}\right), 7.00\left(\mathrm{dd}, 1 \mathrm{H},{ }^{3} J(\mathrm{H}-\right.$ $\mathrm{H})=5.0,{ }^{3} J(\mathrm{H}-\mathrm{H})=3.7, \underline{\mathrm{H}}_{4}$ of $\left.\mathrm{C}_{4} \mathrm{CH}_{3} \mathrm{~S}\right), 7.10\left(\mathrm{~d}, 1 \mathrm{H},{ }^{3} J(\mathrm{H}-\mathrm{H})=3.7, \underline{\mathrm{H}}_{3}\right.$ of $\left.\mathrm{C}_{4} \mathrm{CH}_{3} \mathrm{~S}\right), 7.29(\mathrm{~d}$, $1 \mathrm{H},{ }^{3} J(\mathrm{H}-\mathrm{H})=5.0,{ }^{4} J(\mathrm{H}-\mathrm{H})=1.2, \underline{\mathrm{H}_{5}}$ of $\left.\mathrm{C}_{4} \mathrm{CH}_{3} \mathrm{~S}\right) ;{ }^{13} \mathrm{C} \mathrm{NMR}\left(\mathrm{CDCl}_{3}\right): \delta 16.2\left(\mathrm{~d},{ }^{3} J(\mathrm{C}-\mathrm{P})=\right.$ 6.3, $\left.\underline{\mathrm{CH}}_{3} \mathrm{CH}_{2} \mathrm{O}_{\mathrm{A}}\right), 16.2\left(\mathrm{~d},{ }^{3} J(\mathrm{C}-\mathrm{P})=5.8, \underline{\mathrm{CH}}_{3} \mathrm{CH}_{2} \mathrm{O}_{\mathrm{B}}\right), 24.6\left(\mathrm{t},{ }^{3} J(\mathrm{C}-\mathrm{P})=2.2, \underline{\mathrm{CH}}_{3}\right), 64.9(\mathrm{~d}$, $\left.{ }^{2} J(\mathrm{C}-\mathrm{P})=6.5, \mathrm{CH}_{3} \underline{\mathrm{CH}}_{2} \mathrm{O}_{\mathrm{A}}\right), 64.9\left(\mathrm{~d},{ }^{2} J(\mathrm{C}-\mathrm{P})=7.1, \mathrm{CH}_{3} \underline{\mathrm{CH}}_{2} \mathrm{O}_{\mathrm{B}}\right), 75.6\left(\mathrm{td},{ }^{2} J(\mathrm{C}-\mathrm{F})=23.2\right.$, $\left.{ }^{2} J(\mathrm{C}-\mathrm{P})=15.0, \mathrm{PCF}_{2} \underline{\mathrm{COH}}\right), 118.8\left(\mathrm{td},{ }^{1} J(\mathrm{C}-\mathrm{F})=273.0,{ }^{1} J(\mathrm{C}-\mathrm{P})=202.9, \mathrm{P}^{-} F_{2} \mathrm{COH}\right), 125.5$ (s, $\underline{\mathrm{C}}_{3}$ of $\left.\mathrm{C}_{4} \mathrm{H}_{3} \mathrm{~S}\right), 125.6\left(\mathrm{~s}, \underline{\mathrm{C}}_{4}\right.$ of $\left.\mathrm{C}_{4} \mathrm{H}_{3} \mathrm{~S}\right), 126.8\left(\mathrm{~s}, \underline{\mathrm{C}}_{5}\right.$ of $\left.\mathrm{C}_{4} \mathrm{H}_{3} \mathrm{~S}\right), 145.2\left(\mathrm{~s}, \underline{\mathrm{C}}_{2}\right.$ of $\left.\mathrm{C}_{4} \mathrm{H}_{3} \mathrm{~S}\right)$.

$\mathrm{m} / \mathrm{z}$ (EI) $314\left(\mathrm{M}^{+}, 22 \%\right), 188$ (66), 161 (95), 127 (100).

4.8. General procedure for the transposition of the diethyl 1,1-difluoro 2-hydroxyethyl phosphonates 7 and 8 into 9 and $\mathbf{1 0 .}$

Sodium hydride $(0.15 \mathrm{~g}$ of $60 \%$ dispersion in mineral oil, $3.3 \mathrm{mmol})$ was washed with hexane ( $3 \times 10 \mathrm{ml}$ ) in a three-necked round bottom flask equipped with a thermometer, reflux condenser and an addition funnel and flushed with nitrogen. Magnetic stirring was initiated and THF $(20 \mathrm{ml})$ was added. The suspension was cooled to $0^{\circ} \mathrm{C}$, diethyl 1,1-difluoro-2hydroxyethyl phosphonate (7 or $\mathbf{8}, 3 \mathrm{mmol}$ ) was then added dropwise. After an additional 30 min at $0^{\circ} \mathrm{C}$, a ice-cold mixture of water $(10 \mathrm{ml})$ and brine $(10 \mathrm{ml})$ was added. The aqueous layer was extracted with $\mathrm{CH}_{2} \mathrm{Cl}_{2}(3 \times 10 \mathrm{~mL})$. The extracts were dried $\left(\mathrm{MgSO}_{4}\right)$ and the solvents were removed under reduced pressure to give the crude product ( 9 or $\mathbf{1 0}$ ) which was purified by chromatography.

\subsubsection{Diethyl 1-heptyl 2,2-difluoroethylphosphate $9 a$}

${ }^{31} \mathrm{P}$ NMR $\left(\mathrm{CDCl}_{3}\right): \delta-3.4(\mathrm{~s}) ;{ }^{1} \mathrm{H} \mathrm{NMR}\left(\mathrm{CDCl}_{3}\right): \delta 0.85\left(\mathrm{t}, 3 \mathrm{H},{ }^{3} J(\mathrm{H}-\mathrm{H})=6.4\right.$, $\left.\left(\mathrm{CH}_{2}\right)_{6} \underline{\mathrm{C}}_{3}\right), 1.28\left(\mathrm{~m}, 14 \mathrm{H},\left(\mathrm{C}_{2}\right)_{4} \mathrm{CH}_{3}\right.$ and $\left.\mathrm{C}_{3} \mathrm{CH}_{2} \mathrm{O}\right), 1.47\left(\mathrm{~m}, 2 \mathrm{H}, \mathrm{CH}(\mathrm{OP}) \mathrm{CH}_{2} \mathrm{C}_{2}\right)$, $1.68\left(\mathrm{~m}, 2 \mathrm{H}, \mathrm{CH}(\mathrm{OP}) \mathrm{CH}_{2}\right), 4.12\left(\mathrm{qd}, 4 \mathrm{H},{ }^{3} \mathrm{~J}(\mathrm{H}-\mathrm{H})={ }^{3} J(\mathrm{H}-\mathrm{P})=7.2, \mathrm{CH}_{3} \mathrm{CH}_{2} \mathrm{O}\right), 4.45(\mathrm{~m}, 1 \mathrm{H}$, $\left.\mathrm{HF}_{2} \mathrm{C}-\mathrm{C} \underline{\mathrm{H}}\right), 5.80\left(\mathrm{td}, 1 \mathrm{H},{ }^{2} J(\mathrm{H}-\mathrm{F})=55.3,{ }^{3} J(\mathrm{H}-\mathrm{H})=3.4, \underline{\left.\mathrm{HF}_{2} \mathrm{C}\right)} ;{ }^{13} \mathrm{C} \mathrm{NMR}\left(\mathrm{CDCl}_{3}\right): \delta 14.6\right.$ $\left(\mathrm{s},\left(\mathrm{CH}_{2}\right)_{6} \mathrm{CH}_{3}\right), 16.5\left(\mathrm{~d},{ }^{3} J(\mathrm{C}-\mathrm{P})=2.7, \mathrm{CH}_{3} \mathrm{CH}_{2} \mathrm{O}_{\mathrm{A}}\right), 16.6\left(\mathrm{~d},{ }^{3} J(\mathrm{C}-\mathrm{P})=3.0, \underline{\mathrm{CH}}_{3} \mathrm{CH}_{2} \mathrm{O}_{\mathrm{B}}\right)$, $23.2\left(\mathrm{~s}, \underline{\mathrm{CH}} \mathrm{H}_{2}\right), 24.9\left(\mathrm{~s}, \underline{\mathrm{CH}}_{2}\right), 29.1(\mathrm{~s}, \underline{\mathrm{CH}} \mathrm{H}), 29.8\left(\mathrm{~s}, \underline{\mathrm{CH}}_{2}\right), 30.0\left(\mathrm{~s}, \underline{\mathrm{C}} \mathrm{H}_{2}\right), 32.4\left(\mathrm{~s}, \underline{\mathrm{CH}}_{2}\right), 64.7$ $\left(\mathrm{d},{ }^{2} J(\mathrm{C}-\mathrm{P})=6.1, \mathrm{CH}_{3} \underline{\mathrm{CH}}_{2} \mathrm{O}\right), 76.7\left(\mathrm{td},{ }^{2} J(\mathrm{C}-\mathrm{F})=25.1,{ }^{2} J(\mathrm{C}-\mathrm{P})=5.8, \mathrm{HF}_{2} \mathrm{C}-\underline{\mathrm{CH}}\right), 114.7(\mathrm{td}$, $\left.{ }^{1} J(\mathrm{C}-\mathrm{F})=245.0,{ }^{3} J(\mathrm{C}-\mathrm{P})=4.9, \mathrm{HF}_{2} \mathrm{C}\right)$.

$\mathrm{m} / \mathrm{z}(\mathrm{CI}+\mathrm{ve}) 317\left(\mathrm{M}+\mathrm{H}^{+}, 100 \%\right)$.

4.8.2. Diethyl 1-(1'-methyl)propyl 2,2-difluoroethylphosphate (two diastereomers) $9 b$ 
${ }^{31} \mathrm{P} \mathrm{NMR}\left(\mathrm{CDCl}_{3}\right): \delta$-3.2 (s, maj.), -3.3 (s, min.) ; ${ }^{1} \mathrm{H}$ NMR $\left(\mathrm{CDCl}_{3}\right): \delta 0.92\left(\mathrm{t}, 3 \mathrm{H},{ }^{3} J(\mathrm{H}-\right.$ $\left.\mathrm{H})=7.3, \mathrm{CH}_{2}-\underline{\mathrm{C}}_{3} \min .\right), 0.94\left(\mathrm{t}, 3 \mathrm{H},{ }^{3} J(\mathrm{H}-\mathrm{H})=7.3, \mathrm{CH}_{2}-\underline{\mathrm{C}}_{3}\right.$ maj. $), 0.98\left(\mathrm{~d}, 3 \mathrm{H},{ }^{3} J(\mathrm{H}-\mathrm{H})=\right.$ 6.9, $\mathrm{CH}-\mathrm{CH}_{3}$ maj.), $1.02\left(\mathrm{~d}, 3 \mathrm{H},{ }^{3} \mathrm{~J}(\mathrm{H}-\mathrm{H})=7.0, \mathrm{CH}-\mathrm{CH}_{3} \mathrm{~min}.\right), 1.3\left(\mathrm{~m}_{\text {masked }}, 1 \mathrm{H}, \mathrm{C} \underline{H H}_{-} \mathrm{CH}_{3}\right)$, $1.32\left(\mathrm{t}, 6 \mathrm{H},{ }^{3} J(\mathrm{H}-\mathrm{H})=7.1, \mathrm{C}_{3} \mathrm{CH}_{2} \mathrm{O}\right), 1.54\left(\mathrm{~m}, 1 \mathrm{H}, \mathrm{CH} \underline{\mathrm{H}}-\mathrm{CH}_{3}\right), 1.83\left(\mathrm{~m}, 1 \mathrm{H}, \mathrm{C} \underline{\mathrm{H}}-\mathrm{CH}_{3}\right)$, $4.12\left(\mathrm{p}, 4 \mathrm{H},{ }^{3} J(\mathrm{H}-\mathrm{H})={ }^{3} J(\mathrm{H}-\mathrm{P})=7.2, \mathrm{CH}_{3} \mathrm{CH}_{2} \mathrm{O}\right), 4.4\left(\mathrm{~m}, 1 \mathrm{H}, \mathrm{HF}_{2} \mathrm{C}-\mathrm{C} \underline{\mathrm{H}}\right), 5.81(\mathrm{td}, 1 \mathrm{H}$, $\left.{ }^{2} J(\mathrm{H}-\mathrm{F})=55.2,{ }^{3} J(\mathrm{H}-\mathrm{H})=4.7, \underline{\mathrm{HF}}_{2} \mathrm{C}_{\text {maj. }}\right), 5.85\left(\mathrm{td}, 1 \mathrm{H},{ }^{2} J(\mathrm{H}-\mathrm{F})=54.8,{ }^{3} J(\mathrm{H}-\mathrm{H})=3.9\right.$, $\underline{\mathrm{HF}}_{2} \mathrm{C}_{\min }$ ) ; ${ }^{13} \mathrm{C} \mathrm{NMR}\left(\mathrm{CDCl}_{3}\right): \delta 11.1$ (s, $\mathrm{CH}_{2} \underline{\mathrm{CH}_{3}}$ min.), 11.5 (s, $\mathrm{CH}_{2} \underline{\mathrm{CH}}_{3}$ maj.), 13.4 (s, $\mathrm{CH} \underline{\mathrm{H}}_{3}$ maj.), 14.4 (s, $\mathrm{CH}_{\mathrm{CH}} \mathrm{H}_{3}$ m. $), 15.9$ (d, $\left.{ }^{3} J(\mathrm{C}-\mathrm{P})=3.4, \underline{\mathrm{CH}}_{3} \mathrm{CH}_{2} \mathrm{O}_{\mathrm{A}}\right), 16.0\left(\mathrm{~d},{ }^{3} J(\mathrm{C}-\mathrm{P})=\right.$ 4.4, $\left.\underline{\mathrm{CH}}_{3} \mathrm{CH}_{2} \mathrm{O}_{\mathrm{B}}\right), 24.0$ (s, $\underline{\mathrm{CH}}_{2} \mathrm{CH}_{3}$ min.), 25.8 (s, $\underline{\mathrm{CH}}_{2} \mathrm{CH}_{3}$ maj.), 35.2 (q, ${ }^{3} J(\mathrm{C}-\mathrm{F})={ }^{3} J(\mathrm{C}-\mathrm{P})=$ $3.0, \underline{\mathrm{CHCH}}_{3}$ maj.), $35.6\left(\mathrm{q},{ }^{3} J(\mathrm{C}-\mathrm{F})={ }^{3} J(\mathrm{C}-\mathrm{P})=3.0, \underline{\mathrm{CHCH}}_{3} \min .\right), 64.0\left(\mathrm{~d},{ }^{2} J(\mathrm{C}-\mathrm{P})=5.9\right.$, $\left.\mathrm{CH}_{3} \underline{\mathrm{CH}}_{2} \mathrm{O}\right), 78.5\left(\mathrm{td},{ }^{2} J(\mathrm{C}-\mathrm{F})=24.1,{ }^{2} J(\mathrm{C}-\mathrm{P})=5.9, \mathrm{HF}_{2} \mathrm{C}-\underline{\mathrm{CH}}_{\text {maj }}\right), 79.5\left(\mathrm{td},{ }^{2} J(\mathrm{C}-\mathrm{F})=23.4\right.$, $\left.{ }^{2} J(\mathrm{C}-\mathrm{P})=5.9, \mathrm{HF}_{2} \mathrm{C}-\underline{\mathrm{CH}}_{\min }\right), 114.2\left(\mathrm{td},{ }^{1} J(\mathrm{C}-\mathrm{F})=244.4,{ }^{3} J(\mathrm{C}-\mathrm{P})=4.8, \mathrm{HF}_{2} \underline{\mathrm{C}}_{\min }\right), 114.4$ $\left(\mathrm{td},{ }^{1} J(\mathrm{C}-\mathrm{F})=244.5,{ }^{3} \mathrm{~J}(\mathrm{C}-\mathrm{P})=4.5, \mathrm{HF}_{2} \mathrm{C}_{\text {maj }}.\right)$.

$\mathrm{m} / \mathrm{z}(\mathrm{CI}+\mathrm{ve}) 275\left(\mathrm{M}+\mathrm{H}^{+}, 100 \%\right)$.

\subsubsection{Diethyl 1-(1',1'-dimethyl)ethyl 2,2-difluoroethylphosphate 9c}

${ }^{31} \mathrm{P}$ NMR $\left(\mathrm{CDCl}_{3}\right): \delta-3.2(\mathrm{~s}) ;{ }^{19} \mathrm{~F} \mathrm{NMR}\left(\mathrm{CDCl}_{3}\right): \delta-123.7$ (ddd, A part of ABXY system, $\left.{ }^{2} J(\mathrm{~F}-\mathrm{F})=289.4,{ }^{2} J(\mathrm{~F}-\mathrm{H})=53.3,{ }^{3} J(\mathrm{~F}-\mathrm{H})=11.4, \mathrm{H}_{\mathrm{X}} \mathrm{F}_{\mathrm{A}} \mathrm{F}_{\mathrm{B}} \mathrm{CCH}_{\mathrm{Y}}\right),-125.6(\mathrm{ddd}, \mathrm{B}$ part of $\mathrm{ABXY}$ system, $\left.{ }^{2} J(\mathrm{~F}-\mathrm{F})=289.4,{ }^{2} J(\mathrm{~F}-\mathrm{H})=53.3,{ }^{3} J(\mathrm{~F}-\mathrm{H})=11.4, \mathrm{HXF}_{\mathrm{A}} \mathrm{F}_{\mathrm{B}} \mathrm{CCH}_{\mathrm{Y}}\right) ;{ }^{1} \mathrm{H} \mathrm{NMR}$ $\left(\mathrm{CDCl}_{3}\right): \delta 1.04\left(\mathrm{~s}, 9 \mathrm{H}, \mathrm{C}\left(\mathrm{CH}_{3}\right)_{3}\right), 1.33\left(\mathrm{t}, 6 \mathrm{H},{ }^{3} J(\mathrm{H}-\mathrm{H})=7.1, \mathrm{CH}_{3} \mathrm{CH}_{2} \mathrm{O}\right), 4.14\left(\mathrm{p}, 4 \mathrm{H},{ }^{3} J(\mathrm{H}-\right.$ $\left.\mathrm{H})={ }^{3} J(\mathrm{H}-\mathrm{P})=7.1, \mathrm{CH}_{3} \mathrm{CH}_{2} \mathrm{O}\right), 4.23\left(\mathrm{dddd}, 1 \mathrm{H},{ }^{3} J(\mathrm{H}-\mathrm{P})=15.9,{ }^{3} J(\mathrm{H}-\mathrm{F})=10.3,{ }^{3} J(\mathrm{H}-\mathrm{F})=\right.$ $\left.9.6,{ }^{3} J(\mathrm{H}-\mathrm{H})=2.8, \mathrm{HF}_{2} \mathrm{C}-\mathrm{C} \underline{\mathrm{H}}\right), 5.88\left(\mathrm{td}, 1 \mathrm{H},{ }^{2} J(\mathrm{H}-\mathrm{F})=54.0,{ }^{3} J(\mathrm{H}-\mathrm{H})=2.8, \underline{\mathrm{HF}}_{2} \mathrm{C}\right) ;{ }^{13} \mathrm{C}$ NMR $\left(\mathrm{CDCl}_{3}\right): \delta 15.7\left(\mathrm{~d},{ }^{3} \mathrm{~J}(\mathrm{C}-\mathrm{P})=5.0, \underline{\mathrm{CH}}_{3} \mathrm{CH}_{2} \mathrm{O}_{\mathrm{A}}\right), 15.8\left(\mathrm{~d},{ }^{3} \mathrm{~J}(\mathrm{C}-\mathrm{P})=5.0, \underline{\mathrm{CH}}_{3} \mathrm{CH}_{2} \mathrm{O}_{\mathrm{B}}\right)$, $25.8\left(\mathrm{~s}, \mathrm{C}\left(\underline{\mathrm{CH}}_{3}\right)_{3}\right), 33.6\left(\mathrm{t},{ }^{3} J(\mathrm{C}-\mathrm{F})=3.4, \underline{\mathrm{C}}\left(\mathrm{CH}_{3}\right)_{3}\right), 63.7\left(\mathrm{~d},{ }^{2} J(\mathrm{C}-\mathrm{P})=6.0, \mathrm{CH}_{3} \underline{\mathrm{CH}}_{2} \mathrm{O}\right), 82.4$ $\left(\mathrm{ddd},{ }^{2} J(\mathrm{C}-\mathrm{F})=21.4,{ }^{2} J(\mathrm{C}-\mathrm{F})=18.7,{ }^{2} J(\mathrm{C}-\mathrm{P})=6.1, \mathrm{HF}_{2} \mathrm{C}-\underline{\mathrm{C}} \mathrm{H}\right), 113.8\left(\mathrm{ddd},{ }^{1} J(\mathrm{C}-\mathrm{F})=246.3\right.$, $\left.{ }^{1} J(\mathrm{C}-\mathrm{F})=243.5,{ }^{3} J(\mathrm{C}-\mathrm{P})=2.7, \mathrm{HF}_{2} \mathrm{C}\right)$.

$\mathrm{m} / \mathrm{z}(\mathrm{CI}+\mathrm{ve}) 275\left(\mathrm{M}+\mathrm{H}^{+}, 100 \%\right)$.

\subsubsection{Diethyl 1-(4'-fluoro)phenyl 2,2-difluoroethylphosphate $9 \boldsymbol{e}$}

${ }^{31} \mathrm{P}$ NMR $\left(\mathrm{CDCl}_{3}\right): \delta-3.6(\mathrm{~s}) ;{ }^{19} \mathrm{~F}$ NMR $\left(\mathrm{CDCl}_{3}\right): \delta-112.6\left(\mathrm{~s}, \mathrm{C}_{6} \mathrm{H}_{5} \mathrm{~F}\right),-127.5(\mathrm{ddd}, \mathrm{A}$ part of $\mathrm{ABXY}$ system, $\left.{ }^{2} J(\mathrm{~F}-\mathrm{F})=285.6,{ }^{2} J(\mathrm{~F}-\mathrm{H})=53.3,{ }^{3} J(\mathrm{~F}-\mathrm{H})=11.4, \mathrm{H}_{\mathrm{X}} \mathrm{F}_{\mathrm{A}} \mathrm{F}_{\mathrm{B}} \mathrm{CCHY}\right)$, $130.3\left(\mathrm{ddd}, \mathrm{B}\right.$ part of ABXY system, ${ }^{2} J(\mathrm{~F}-\mathrm{F})=285.6,{ }^{2} J(\mathrm{~F}-\mathrm{H})=53.3,{ }^{3} J(\mathrm{~F}-\mathrm{H})=11.4$, $\left.\mathrm{H}_{\mathrm{X}} \mathrm{F}_{\mathrm{A}} \mathrm{F}_{\mathrm{B}} \mathrm{CCH}_{\mathrm{Y}}\right) ;{ }^{1} \mathrm{H} \mathrm{NMR}\left(\mathrm{CDCl}_{3}\right): \delta 1.17\left(\mathrm{t}, 3 \mathrm{H},{ }^{3} \mathrm{~J}(\mathrm{H}-\mathrm{H})=7.1, \mathrm{CH}_{3} \mathrm{CH}_{2} \mathrm{O}_{\mathrm{A}}\right), 1.28(\mathrm{t}, 3 \mathrm{H}$, $\left.{ }^{3} J(\mathrm{H}-\mathrm{H})=7.1, \mathrm{CH}_{3} \mathrm{CH}_{2} \mathrm{O}_{\mathrm{B}}\right), 3.94\left(\mathrm{pd}, 2 \mathrm{H},{ }^{3} J(\mathrm{H}-\mathrm{H})={ }^{3} J(\mathrm{H}-\mathrm{P})=7.1, J=2.5, \mathrm{CH}_{3} \mathrm{C}_{2} \mathrm{O}_{\mathrm{A}}\right)$, $4.09\left(\mathrm{q}, 2 \mathrm{H},{ }^{3} J(\mathrm{H}-\mathrm{H})=7.1, \mathrm{CH}_{3} \mathrm{CH}_{2} \mathrm{OB}_{\mathrm{B}}\right), 5.36\left(\mathrm{qd}, 1 \mathrm{H},{ }^{3} J(\mathrm{H}-\mathrm{P})={ }^{3} J(\mathrm{H}-\mathrm{F})=10.1,{ }^{3} J(\mathrm{H}-\mathrm{H})=\right.$ $\left.4.0, \mathrm{HF}_{2} \mathrm{C}-\mathrm{C} \underline{\mathrm{H}}\right), 5.88\left(\mathrm{td}, 1 \mathrm{H},{ }^{2} J(\mathrm{H}-\mathrm{F})=55.2,{ }^{3} J(\mathrm{H}-\mathrm{H})=4.0, \underline{\mathrm{HF}}_{2} \mathrm{C}\right), 7.09\left(\mathrm{t}, 2 \mathrm{H},{ }^{3} J(\mathrm{H}-\mathrm{H})=\right.$ ${ }^{3} J(\mathrm{H}-\mathrm{F})=8.7, \underline{\mathrm{H}}_{\text {meta }}$ of $\left.\mathrm{C}_{6} \mathrm{H}_{4} \mathrm{~F}\right), 7.41\left(\mathrm{dd}, 2 \mathrm{H},{ }^{3} J(\mathrm{H}-\mathrm{H})={ }^{3} J(\mathrm{H}-\mathrm{F})=8.7,{ }^{3} J(\mathrm{H}-\mathrm{F})=5.3, \underline{\mathrm{H}}_{\text {ortho }}\right.$ 
of $\left.\mathrm{C}_{6} \mathrm{H}_{4} \mathrm{~F}\right) ;{ }^{13} \mathrm{C}$ NMR $\left(\mathrm{CDCl}_{3}\right): \delta 16.2\left(\mathrm{~d},{ }^{3} J(\mathrm{C}-\mathrm{P})=3.0, \underline{\mathrm{CH}}_{3} \mathrm{CH}_{2} \mathrm{O}_{\mathrm{A}}\right), 16.3\left(\mathrm{~d},{ }^{3} J(\mathrm{C}-\mathrm{P})=\right.$ 3.8, $\left.\underline{\mathrm{CH}}_{3} \mathrm{CH}_{2} \mathrm{O}_{\mathrm{B}}\right), 64.6\left(\mathrm{~d},{ }^{2} J(\mathrm{C}-\mathrm{P})=6.0, \mathrm{CH}_{3} \underline{\mathrm{CH}_{2} \mathrm{O}_{\mathrm{A}}}\right), 64.8\left(\mathrm{~d},{ }^{2} J(\mathrm{C}-\mathrm{P})=6.2, \mathrm{CH}_{3} \underline{\mathrm{CH}}_{2} \mathrm{O}_{\mathrm{B}}\right)$, $77.1\left(\mathrm{td},{ }^{2} J(\mathrm{C}-\mathrm{F})=26.6,{ }^{2} J(\mathrm{C}-\mathrm{P})=4.7, \mathrm{HF}_{2} \mathrm{C}-\underline{\mathrm{CH}}\right), 114.1\left(\mathrm{td},{ }^{1} J(\mathrm{C}-\mathrm{F})=246.6,{ }^{3} J(\mathrm{C}-\mathrm{P})=8.7\right.$, $\left.\mathrm{HF}_{2} \underline{\mathrm{C}}\right), 116.2\left(\mathrm{~d},{ }^{3} J(\mathrm{C}-\mathrm{F})=22.2, \underline{\mathrm{C}}_{\text {meta }}\right.$ of $\left.\mathrm{C}_{6} \mathrm{H}_{4} \mathrm{~F}\right), 129.2\left(\mathrm{~s}_{\text {large }}, \underline{\mathrm{C}}_{\text {ipso }}\right.$ of $\left.\mathrm{C}_{6} \mathrm{H}_{4} \mathrm{~F}\right), 130.2(\mathrm{~d}$, ${ }^{4} J(\mathrm{C}-\mathrm{F})=8.2, \underline{\mathrm{C}}_{\text {ortho }}$ of $\left.\mathrm{C}_{6} \mathrm{H}_{4} \mathrm{~F}\right), 163.8\left(\mathrm{~d},{ }^{1} J(\mathrm{C}-\mathrm{F})=248.7, \underline{\mathrm{C}}_{\text {para }}\right.$ of $\left.\mathrm{C}_{6} \mathrm{H}_{4} \mathrm{~F}\right)$.

$\mathrm{m} / \mathrm{z}$ (EI) $313\left(\mathrm{M}+\mathrm{H}^{+}, 1 \%\right), 292$ (44), 244 (55), 216 (84).

\subsubsection{Diethyl 1-(4'-methyl)phenyl 2,2-difluoroethylphosphate $9 f$}

${ }^{31} \mathrm{P}$ NMR $\left(\mathrm{CDCl}_{3}\right): \delta-3.8(\mathrm{~s}) ;{ }^{1} \mathrm{H}$ NMR $\left(\mathrm{CDCl}_{3}\right): \delta 1.16\left(\mathrm{t}, 3 \mathrm{H},{ }^{3} J(\mathrm{H}-\mathrm{H})=7.1\right.$, $\left.\mathrm{C}_{3} \mathrm{CH}_{2} \mathrm{O}_{\mathrm{A}}\right), 1.27\left(\mathrm{t}, 3 \mathrm{H},{ }^{3} \mathrm{~J}(\mathrm{H}-\mathrm{H})=7.1, \mathrm{CH}_{3} \mathrm{CH}_{2} \mathrm{O}_{\mathrm{B}}\right), 2.35\left(\mathrm{~s}, 3 \mathrm{H}, \mathrm{C}_{6} \mathrm{H}_{4} \mathrm{C}_{3}\right), 3.92(\mathrm{pd}, 2 \mathrm{H}$, $\left.{ }^{3} J(\mathrm{H}-\mathrm{H})={ }^{3} J(\mathrm{H}-\mathrm{P})=7.1, J=3.2, \mathrm{CH}_{3} \underline{\mathrm{H}}_{2} \mathrm{O}_{\mathrm{A}}\right), 4.10\left(\mathrm{q}, 2 \mathrm{H},{ }^{3} J(\mathrm{H}-\mathrm{H})=7.1, \mathrm{CH}_{3} \mathrm{CH}_{2} \mathrm{O}_{\mathrm{B}}\right)$, $5.33\left(\mathrm{qd}, 1 \mathrm{H},{ }^{3} J(\mathrm{H}-\mathrm{P})={ }^{3} J(\mathrm{H}-\mathrm{F})=10.0,{ }^{3} J(\mathrm{H}-\mathrm{H})=4.2, \mathrm{HF}_{2} \mathrm{C}-\mathrm{C} \underline{\mathrm{H}}\right), 5.87\left(\mathrm{td}, 1 \mathrm{H},{ }^{2} J(\mathrm{H}-\mathrm{F})=\right.$ $\left.55.3,{ }^{3} J(\mathrm{H}-\mathrm{H})=4.2, \underline{H F}_{2} \mathrm{C}\right), 7.20\left(\mathrm{~d}, 2 \mathrm{H},{ }^{3} J(\mathrm{H}-\mathrm{H})=7.9, \underline{\mathrm{H}}_{\text {meta }}\right.$ of $\left.\mathrm{C}_{6} \mathrm{H}_{4} \mathrm{CH}_{3}\right), 7.30(\mathrm{~d}, 2 \mathrm{H}$, ${ }^{3} J(\mathrm{H}-\mathrm{H})=7.9, \underline{\mathrm{H}}_{\text {ortho }}$ of $\left.\mathrm{C}_{6} \mathrm{H}_{4} \mathrm{CH}_{3}\right) ;{ }^{13} \mathrm{C} \mathrm{NMR}\left(\mathrm{CDCl}_{3}\right): \delta 15.7\left(\mathrm{~d},{ }^{3} J(\mathrm{C}-\mathrm{P})=4.8\right.$, $\left.\mathrm{CH}_{3} \mathrm{CH}_{2} \mathrm{O}_{\mathrm{A}}\right), 15.8\left(\mathrm{~d},{ }^{3} J(\mathrm{C}-\mathrm{P})=3.6, \mathrm{CH}_{3} \mathrm{CH}_{2} \mathrm{O}_{\mathrm{B}}\right), 21.1\left(\mathrm{~s}, \mathrm{C}_{6} \mathrm{H}_{4} \mathrm{CH}_{3}\right), 64.1\left(\mathrm{~d},{ }^{2} J(\mathrm{C}-\mathrm{P})=\right.$ $\left.6.1, \mathrm{CH}_{3} \underline{\mathrm{CH}}_{2} \mathrm{O}_{\mathrm{A}}\right), 64.2\left(\mathrm{~d},{ }^{2} J(\mathrm{C}-\mathrm{P})=6.1, \mathrm{CH}_{3} \underline{\mathrm{CH}}_{2} \mathrm{O}_{\mathrm{B}}\right), 77.5\left(\mathrm{td},{ }^{2} J(\mathrm{C}-\mathrm{F})=26.2,{ }^{2} J(\mathrm{C}-\mathrm{P})=\right.$ $\left.5.0, \mathrm{HF}_{2} \mathrm{C}-\underline{\mathrm{CH}}\right), 114.1\left(\mathrm{td},{ }^{1} J(\mathrm{C}-\mathrm{F})=245.8,{ }^{3} J(\mathrm{C}-\mathrm{P})=9.0, \mathrm{HF}_{2} \mathrm{C}\right), 127.7$ (s, $\underline{\mathrm{C}}_{\text {ortho }}$ of $\mathrm{C}_{6} \mathrm{H}_{4} \mathrm{CH}_{3}$ ), 129.4 (s, $\underline{\mathrm{C}}_{\text {meta }}$ of $\mathrm{C}_{6} \mathrm{H}_{4} \mathrm{CH}_{3}$ ), 130.1 (s sarge,$\underline{\mathrm{C}}_{\text {ipso }}$ of $\mathrm{C}_{6} \mathrm{H}_{4} \mathrm{CH}_{3}$ ), 139.6 (s, $\underline{\mathrm{C}}_{\text {para }}$ of $\left.\mathrm{C}_{6} \mathrm{H}_{4} \mathrm{CH}_{3}\right)$.

$\mathrm{m} / \mathrm{z}(\mathrm{EI}) 309\left(\mathrm{M}+\mathrm{H}^{+}, 1 \%\right), 288(100)$.

\subsubsection{Diethyl 1-(4'-methoxyphenyl) 2,2-difluoroethylphosphate $9 \mathrm{~g}$}

${ }^{31} \mathrm{P}$ NMR $\left(\mathrm{CDCl}_{3}\right): \delta-3.7(\mathrm{~s}) ;{ }^{1} \mathrm{H}$ NMR $\left(\mathrm{CDCl}_{3}\right): \delta 1.13\left(\mathrm{t}, 3 \mathrm{H},{ }^{3} J(\mathrm{H}-\mathrm{H})=7.1\right.$, $\left.\mathrm{C}_{3} \mathrm{CH}_{2} \mathrm{O}_{\mathrm{A}}\right), 1.24\left(\mathrm{t}, 3 \mathrm{H},{ }^{3} J(\mathrm{H}-\mathrm{H})=7.1, \mathrm{CH}_{3} \mathrm{CH}_{2} \mathrm{O}_{\mathrm{B}}\right), 3.77\left(\mathrm{~s}, 3 \mathrm{H}, \mathrm{C}_{6} \mathrm{H}_{4} \mathrm{OC} \underline{H}_{3}\right), 3.88$ (pd, $\left.2 \mathrm{H},{ }^{3} J(\mathrm{H}-\mathrm{H})={ }^{3} J(\mathrm{H}-\mathrm{P})=7.1, J=3.2, \mathrm{CH}_{3} \mathrm{CH}_{2} \mathrm{O}_{\mathrm{A}}\right), 4.07\left(\mathrm{~m}, 2 \mathrm{H}, \mathrm{CH}_{3} \mathrm{CH}_{2} \mathrm{O}_{\mathrm{B}}\right), 5.30(\mathrm{qd}, 1 \mathrm{H}$, $\left.{ }^{3} J(\mathrm{H}-\mathrm{P})={ }^{3} J(\mathrm{H}-\mathrm{F})=10.0,{ }^{3} J(\mathrm{H}-\mathrm{H})=4.1, \mathrm{HF}_{2} \mathrm{C}-\mathrm{C} \underline{\mathrm{H}}\right), 5.85\left(\mathrm{td}, 1 \mathrm{H},{ }^{2} J(\mathrm{H}-\mathrm{F})=55.3,{ }^{3} J(\mathrm{H}-\right.$ $\left.\mathrm{H})=4.1, \underline{\mathrm{HF}}_{2} \mathrm{C}\right), 6.88\left(\mathrm{~d}, 2 \mathrm{H},{ }^{3} J(\mathrm{H}-\mathrm{H})=8.7, \underline{\mathrm{H}}_{\text {meta }}\right.$ of $\left.\mathrm{C}_{6} \mathrm{H}_{4} \mathrm{OCH}_{3}\right), 7.32\left(\mathrm{~d}, 2 \mathrm{H},{ }^{3} J(\mathrm{H}-\mathrm{H})=\right.$ 8.7, $\underline{\mathrm{H}}_{\mathrm{ortho}}$ of $\left.\mathrm{C}_{6} \mathrm{H}_{4} \mathrm{OCH}_{3}\right) ;{ }^{13} \mathrm{C} \mathrm{NMR}\left(\mathrm{CDCl}_{3}\right): \delta 15.7\left(\mathrm{~d},{ }^{3} J(\mathrm{C}-\mathrm{P})=4.2, \underline{\mathrm{CH}}_{3} \mathrm{CH}_{2} \mathrm{O}_{\mathrm{A}}\right), 15.8$ $\left(\mathrm{d},{ }^{3} J(\mathrm{C}-\mathrm{P})=4.5, \underline{\mathrm{CH}}_{3} \mathrm{CH}_{2} \mathrm{O}_{\mathrm{B}}\right), 55.1\left(\mathrm{~s}, \mathrm{C}_{6} \mathrm{H}_{4} \mathrm{OCH}_{3}\right), 64.0\left(\mathrm{~d},{ }^{2} J(\mathrm{C}-\mathrm{P})=6.9, \mathrm{CH}_{3} \underline{\mathrm{CH}}_{2} \mathrm{O}_{\mathrm{A}}\right)$, $64.1\left(\mathrm{~d},{ }^{2} J(\mathrm{C}-\mathrm{P})=7.0, \mathrm{CH}_{3} \underline{\mathrm{CH}}_{2} \mathrm{O}_{\mathrm{B}}\right), 77.2\left(\mathrm{td},{ }^{2} J(\mathrm{C}-\mathrm{F})=26.2,{ }^{2} J(\mathrm{C}-\mathrm{P})=5.0, \mathrm{HF}_{2} \mathrm{C}-\underline{\mathrm{C}} \mathrm{H}\right)$, $114.1\left(\mathrm{~s}, \underline{\mathrm{C}}_{\text {meta }}\right.$ of $\left.\mathrm{C}_{6} \mathrm{H}_{4} \mathrm{OCH}_{3}\right), 114.1\left(\mathrm{td},{ }^{1} J(\mathrm{C}-\mathrm{F})=245.9,{ }^{3} J(\mathrm{C}-\mathrm{P})=9.2, \mathrm{HF}_{2} \mathrm{C}\right), 125.0(\mathrm{~d}$, ${ }^{3} J(\mathrm{C}-\mathrm{P})=2.0, \underline{\mathrm{C}}_{\mathrm{ipso}}$ of $\left.\mathrm{C}_{6} \mathrm{H}_{4} \mathrm{OCH}_{3}\right), 129.3\left(\mathrm{~s}, \underline{\mathrm{C}}_{\text {ortho }}\right.$ of $\left.\mathrm{C}_{6} \mathrm{H}_{4} \mathrm{OCH}_{3}\right), 160.7$ (s, $\underline{\mathrm{C}}_{\text {para }}$ of $\left.\mathrm{C}_{6} \mathrm{H}_{4} \mathrm{OCH}_{3}\right)$.

$\mathrm{m} / \mathrm{z}$ (EI) $324\left(\mathrm{M}^{+}, 3 \%\right), 304(100)$. 
${ }^{31} \mathrm{P} \mathrm{NMR}\left(\mathrm{CDCl}_{3}\right): \delta \quad-4.0(\mathrm{~s}) ;{ }^{1} \mathrm{H}$ NMR $\left(\mathrm{CDCl}_{3}\right): \delta 1.31\left(\mathrm{q}, 6 \mathrm{H},{ }^{3} J(\mathrm{H}-\mathrm{H})=7.1\right.$, $\mathrm{CH}_{3} \mathrm{CH}_{2} \mathrm{O}_{\mathrm{A}}$ and $\left.\mathrm{B}\right), 4.1\left(\mathrm{~m}, 2 \mathrm{H}, \mathrm{CH}_{3} \underline{\mathrm{C}}_{2} \mathrm{O}_{\mathrm{A}}\right), 4.21\left(\mathrm{p}, 2 \mathrm{H},{ }^{3} J(\mathrm{H}-\mathrm{H})={ }^{3} J(\mathrm{H}-\mathrm{P})=7.1\right.$, $\left.\mathrm{CH}_{3} \mathrm{CH}_{2} \mathrm{O}_{\mathrm{B}}\right), 5.52\left(\mathrm{qd}, 1 \mathrm{H},{ }^{3} J(\mathrm{H}-\mathrm{P})={ }^{3} J(\mathrm{H}-\mathrm{F})=10.3,{ }^{3} J(\mathrm{H}-\mathrm{H})=3.6, \mathrm{HF}_{2} \mathrm{C}-\mathrm{C} \underline{\mathrm{H}}\right), 6.22(\mathrm{td}$, $\left.1 \mathrm{H},{ }^{2} J(\mathrm{H}-\mathrm{F})=54.7,{ }^{3} J(\mathrm{H}-\mathrm{H})=3.6, \underline{H F}_{2} \mathrm{C}\right), 7.30\left(\mathrm{dd}, 1 \mathrm{H},{ }^{3} J(\mathrm{H}-\mathrm{H})=7.7\right.$ and $4.8, \underline{\mathrm{H}}_{5}{ }^{\prime}$ of $\left.\mathrm{C}_{5} \mathrm{H}_{4} \mathrm{~N}\right), 7.53\left(\mathrm{~d}_{\text {large }}, 1 \mathrm{H},{ }^{3} J(\mathrm{H}-\mathrm{H})=7.7, \underline{\mathrm{H}}_{3}\right.$ of $\left.\mathrm{C}_{5} \mathrm{H}_{4} \mathrm{~N}\right), 7.76\left(\mathrm{td}, 1 \mathrm{H},{ }^{3} J(\mathrm{H}-\mathrm{H})=7.7,{ }^{4} J(\mathrm{H}-\right.$ $\mathrm{H})=1.8, \underline{\mathrm{H}}_{4}$ of $\left.\mathrm{C}_{5} \mathrm{H}_{4} \mathrm{~N}\right), 8.61\left(\mathrm{dd}, 1 \mathrm{H},{ }^{3} J(\mathrm{H}-\mathrm{H})=4.8,{ }^{4} J(\mathrm{H}-\mathrm{H})=1.8, \underline{\mathrm{H}}_{6}{ }^{\prime}\right.$ of $\left.\mathrm{C}_{5} \mathrm{H}_{4} \mathrm{~N}\right) ;{ }^{13} \mathrm{C}$ NMR $\left(\mathrm{CDCl}_{3}\right): \delta 15.8\left(\mathrm{t},{ }^{3} J(\mathrm{C}-\mathrm{P})=7.0\right.$ and $6.0, \underline{\mathrm{CH}}_{3} \mathrm{CH}_{2} \mathrm{O}_{\mathrm{A}}$ and $\left.\mathrm{B}\right), 64.3\left(\mathrm{t},{ }^{2} J(\mathrm{C}-\mathrm{P})=6.8\right.$ and

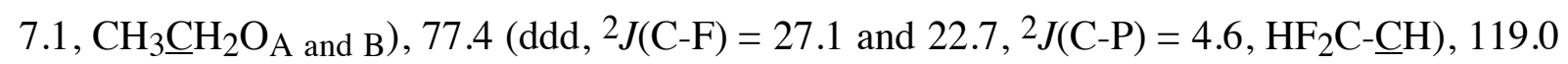
$\left(\mathrm{td},{ }^{1} J(\mathrm{C}-\mathrm{F})=248.2,{ }^{3} J(\mathrm{C}-\mathrm{P})=5.6, \mathrm{HF}_{2} \mathrm{C}\right), 122.7\left(\mathrm{~s}, \underline{\mathrm{C}}_{3^{\prime}}\right.$ of $\left.\mathrm{C}_{5} \mathrm{H}_{4} \mathrm{~N}\right), 124.2\left(\mathrm{~s}, \underline{\mathrm{C}}_{5}{ }^{\prime}\right.$ of $\left.\mathrm{C}_{5} \mathrm{H}_{4} \mathrm{~N}\right)$, $137.2\left(\mathrm{~s}, \underline{\mathrm{C}}_{4^{\prime}}\right.$ of $\left.\mathrm{C}_{5} \mathrm{H}_{4} \mathrm{~N}\right), 149.4\left(\mathrm{~s}, \underline{\mathrm{C}}_{6^{\prime}}\right.$ of $\left.\mathrm{C}_{5} \mathrm{H}_{4} \mathrm{~N}\right), 153.0\left(\mathrm{~d},{ }^{3} J(\mathrm{C}-\mathrm{P})=2.8, \underline{\mathrm{C}}_{2}{ }^{\prime}\right.$ of $\left.\mathrm{C}_{5} \mathrm{H}_{4} \mathrm{~N}\right)$.

$\mathrm{m} / \mathrm{z}(\mathrm{EI}) 295\left(\mathrm{M}^{+}, 31 \%\right), 275$ (45), 250 (52), 227 (82).

\subsubsection{Diethyl (1'-difluoromethyl)cyclohexylphosphate 10a}

${ }^{31} \mathrm{P}$ NMR $\left(\mathrm{CDCl}_{3}\right): \delta-7.5(\mathrm{~s}) ;{ }^{19} \mathrm{~F}$ NMR $\left(\mathrm{CDCl}_{3}\right): \delta-133.9\left(\mathrm{~d},{ }^{2} J(\mathrm{~F}-\mathrm{H})=57.1\right.$, $\left.\mathrm{HF}_{2} \mathrm{CCOP}\right) ;{ }^{1} \mathrm{H} \mathrm{NMR}\left(\mathrm{CDCl}_{3}\right): \delta 1.18\left(\mathrm{~m}, 1 \mathrm{H}, \mathrm{COH}\left(\mathrm{CH}_{2} \mathrm{CH}_{2}\right)_{2} \mathrm{CH}_{\mathrm{ax}} \mathrm{H}_{\mathrm{eq}}\right), 1.32(\mathrm{t}, 6 \mathrm{H}$, $\left.{ }^{3} J(\mathrm{H}-\mathrm{H})=7.1, \mathrm{C}_{3} \mathrm{CH}_{2} \mathrm{O}\right), 1.62\left(\mathrm{~m}+\mathrm{d}, 7 \mathrm{H},{ }^{3} J(\mathrm{H}-\mathrm{H})=6.3, \mathrm{COH}\left(\mathrm{C}_{\mathrm{ax}} \mathrm{H}_{\mathrm{eq}} \underline{\mathrm{C}}_{2}\right)_{2} \mathrm{CH}_{\mathrm{ax}} \underline{\mathrm{H}}_{\mathrm{eq}}\right)$, $2.09\left(\mathrm{~d}_{\text {large }}, 2 \mathrm{H},{ }^{3} J(\mathrm{H}-\mathrm{H})=10.5, \mathrm{COH}\left(\mathrm{CH}_{\mathrm{ax}} \underline{\mathrm{H}}_{\mathrm{eq}} \mathrm{CH}_{2}\right)_{2} \mathrm{CH}_{2}\right), 4.10\left(\mathrm{p}, 4 \mathrm{H},{ }^{3} J(\mathrm{H}-\mathrm{H})={ }^{3} J(\mathrm{H}-\right.$ $\left.\mathrm{P})=7.1, \mathrm{CH}_{3} \underline{\mathrm{CH}}_{2} \mathrm{O}\right), 6.12\left(\mathrm{t}, 1 \mathrm{H},{ }^{2} \mathrm{~J}(\mathrm{H}-\mathrm{F})=56.2, \underline{\mathrm{HF}}_{2} \mathrm{C}\right) ;{ }^{13} \mathrm{C} \mathrm{NMR}\left(\mathrm{CDCl}_{3}\right): \delta 16.3(\mathrm{~d}$, $\left.{ }_{3} J(\mathrm{C}-\mathrm{P})=7.5, \mathrm{CH}_{3} \mathrm{CH}_{2} \mathrm{O}\right), 20.8\left(\mathrm{~s}, \mathrm{COH}\left(\mathrm{CH}_{2} \mathrm{CH}_{2}\right)_{2} \mathrm{CH}_{2}\right), 25.3\left(\mathrm{~s}, \mathrm{COH}\left(\mathrm{CH}_{2} \mathrm{CH}_{2}\right)_{2} \mathrm{CH}_{2}\right)$, $29.1\left(\mathrm{t},{ }^{3} \mathrm{~J}(\mathrm{C}-\mathrm{F})=3.1, \mathrm{COH}\left(\underline{\mathrm{CH}}_{2} \mathrm{CH}_{2}\right)_{2} \mathrm{CH}_{2}\right), 64.2\left(\mathrm{~d},{ }^{2} J(\mathrm{C}-\mathrm{P})=6.2, \mathrm{CH}_{3} \underline{\mathrm{CH}}_{2} \mathrm{O}\right), 83.5(\mathrm{td}$, $\left.{ }^{2} J(\mathrm{C}-\mathrm{F})=22.7,{ }^{2} J(\mathrm{C}-\mathrm{P})=7.5, \mathrm{HF}_{2} \mathrm{C}-\underline{\mathrm{CH}}\right), 115.3\left(\mathrm{t},{ }^{1} J(\mathrm{C}-\mathrm{F})=247.3, \mathrm{HF}_{2} \mathrm{C}\right)$.

$\mathrm{m} / \mathrm{z}(\mathrm{CI}+\mathrm{ve}) 287\left(\mathrm{M}+\mathrm{H}^{+}, 100 \%\right)$.

\subsubsection{1,1-Difluoro 2-(4'-dimethylamino)phenyl ethene issued from $7 \boldsymbol{h}$}

${ }^{1} \mathrm{H} \mathrm{NMR}\left(\mathrm{CDCl}_{3}\right): \delta 2.98\left(\mathrm{~s}, 6 \mathrm{H}, \mathrm{N}\left(\mathrm{CH}_{3}\right)_{2}\right), 5.20\left(\mathrm{dd}, 1 \mathrm{H},{ }^{3} J(\mathrm{H}-\mathrm{Ftrans})=26.9,{ }^{3} J(\mathrm{H}-\right.$ Fcis $\left.)=4.0, \mathrm{~F}_{2} \mathrm{C}=\mathrm{C} \underline{\mathrm{H}}\right), 6.72\left(\mathrm{~d}, 2 \mathrm{H},{ }^{3} J(\mathrm{H}-\mathrm{H})=8.8, \underline{\mathrm{H}}_{\text {meta }}\right.$ of $\left.\mathrm{C}_{6} \mathrm{H}_{4}\right), 7.24\left(\mathrm{~d}, 2 \mathrm{H},{ }^{3} J(\mathrm{H}-\mathrm{H})=\right.$ $8.8, \underline{\mathrm{H}}_{\text {ortho }}$ of $\left.\mathrm{C}_{6} \mathrm{H}_{4}\right)$.

$\mathrm{m} / \mathrm{z}(\mathrm{EI}) 183\left(\mathrm{M}^{+}, 100 \%\right), 167$ (44).

\subsubsection{0. (1-Difluoromethylene-1,2,3,4-tetrahydro)naphtyl issued from $8 \mathrm{c}$}

${ }^{1} \mathrm{H} \mathrm{NMR}\left(\mathrm{CDCl}_{3}\right): \delta 1.92\left(\mathrm{pd}, 2 \mathrm{H},{ }^{3} J(\mathrm{H}-\mathrm{H})=6.3,{ }^{5} J(\mathrm{H}-\mathrm{F})=1.6, \underline{\mathrm{H}}_{3}\right), 2.55\left(\mathrm{~m}, 2 \mathrm{H}, \underline{\mathrm{H}}_{2}\right)$, $2.87\left(\mathrm{t}, 2 \mathrm{H},{ }^{3} J(\mathrm{H}-\mathrm{H})=6.3, \underline{\mathrm{H}}_{4}\right), 7.15\left(\mathrm{~m}, 3 \mathrm{H}, \underline{\mathrm{H}}_{5}, 6\right.$ and 7$), 7.65\left(\mathrm{~d}_{\text {large }}, 1 \mathrm{H}{ }^{5} J(\mathrm{H}-\mathrm{F})=6.3, \underline{\mathrm{H}}_{8}\right)$; ${ }^{13} \mathrm{C}$ NMR $\left(\mathrm{CDCl}_{3}\right): \delta 24.0\left(\mathrm{~s}, \underline{\mathrm{C}}_{3}\right), 30.5\left(\mathrm{~s}, \underline{\mathrm{C}}_{4}\right), 31.1\left(\mathrm{~s}, \underline{\mathrm{C}}_{2}\right), 89.0\left(\mathrm{dd},{ }^{2} J(\mathrm{C}-\mathrm{F})=22.4\right.$ and $\left.10.0, \underline{\mathrm{C}}_{1}\right), 126.9$ (s, $\left.\underline{\mathrm{C}}_{6}\right), 127.3\left(\mathrm{~s}, \underline{\mathrm{C}}_{7}\right), 127.9\left(\mathrm{~d},{ }^{4} J(\mathrm{C}-\mathrm{F})=13.9, \underline{\mathrm{C}}_{8}\right), 129.7$ (s, $\underline{\mathrm{C}}_{5}$ and $\left.\underline{\mathrm{C}}_{10}\right)$, $138.2\left(\mathrm{~d},{ }^{3} J(\mathrm{C}-\mathrm{F})=4.0, \underline{\mathrm{C}}_{9}\right), 153.6\left(\mathrm{dd},{ }^{1} J(\mathrm{C}-\mathrm{F})=294.7\right.$ and $\left.285.2,=\underline{\mathrm{CF}}_{2}\right)$.

$\mathrm{m} / \mathrm{z}(\mathrm{EI}) 180\left(\mathrm{M}^{+}, 35\right), 147$ (70), 129 (100). 


\section{Acknowledgments}

We gratefully acknowledge the financial support by the Centre National de la Recherche Scientifique (R. W.). We are also grateful to Miss N. Phung (URA 459) of the University of Reims for ${ }^{19} \mathrm{~F}$ measurements, and Mr. M. Levard (URA 1307) of the Ecole Polytechnique for the mass spectra.

\section{References}

1. W. F. Bailey and J. J. Patricia, J. Organomet. Chem. 352 (1988) 1.

2. (a) D. Seyferth and R. S. Marmor, J. Organomet. Chem. 59 (1973) 237. (b) J. F. Normant, P. Perriot and J. Villieras, Synthesis (1975) 458. (c) P. Coutrot, C. Laurenco, J. F.Normant, P. Perriot, P. Savignac and J. Villieras, Synthesis (1977) 615. (d) J. Villieras, A. Reliquet and J. F. Normant, Synthesis (1978) 27. (e) J. Villieras, P. Perriot and J. F. Normant, Synthesis (1978) 29. (f) J. Villieras, P. Perriot and J. F. Normant, Synthesis (1978) 31. (g) P. Perriot, J. Villieras and J. F. Normant, Synthesis (1978) 33. (h) G. T. Lowen and M. R. Almond, J. Org. Chem. 59 (1994) 4548. (i) C. Grandin, N. Collignon and P. Savignac, Synthesis (1995) 239. (j) Y. Zanella, S. Berté-Verrando, R. Dizière and P. Savignac, J. Chem. Soc. Perkin Trans. I (1995) 2835. (k) R. Dizière and P. Savignac, Tetrahedron Lett. 37 (1996) 1783.

3. A. Marinetti and P. Savignac, Diethyl 1,1-dichloromethylphosphonate. Preparation and use in the syntheses of alkynes Organic Syntheses (in press).

4. (a) D. J. Burton, R. Takei and S. Shin-Ya, J. Fluorine Chem. 18 (1981) 197. (b) D. J. Burton, T. Ishihara and M. Maruta, Chem. Lett. (1982) 755. (c) M. Obayashi, E. Ito, K. Matsui and K. Kondo, Tetrahedron Lett. 23 (1982) 2323. (d) G. M. Blackburn and M. J. Parratt, J. Chem. Soc., Chem. Commun. (1983) 886. (e) D. J. Burton, L. G. Sprague, D. J. Pietrzyk and S. H. Edelmuth, J. Org. Chem. 49 (1984) 3437. (f) G. M. Blackburn, D. Brown and S. J. Martin , J. Chem. Res., Synop. (1985) 92. (g) D. J. Burton and L. G. Sprague, J. Org. Chem. 53 (1988) 1523. (h) D. J. Burton and L. G. Sprague, J. Org. Chem. 54 (1989) 613. (i) R. D. Chambers, R. Jaouhari and D. O'Hagan, J. Fluorine Chem. 44 (1989) 275. (j) L. G. Sprague, D. J. Burton, R. D. Guneratne and W. E. Benett, J. Fluorine Chem. 49 (1990) 75. (k) S. Halazy, A. Ehrhard and C. Danzin, J. Am. Chem. Soc. 113 (1991) 315. (1) S. F. Martin, D. W. Dean and A. S. Wagman, Tetrahedron Lett. 33 (1992) 1839. (m) S. Chen and C. Yuan, Phosphorus, Sulfur, and Silicon 82 (1993) 73. (n) D. B. Berkowitz, M. Eggen, Q. Shen, D. G. Sloss, J. Org. Chem. 58 (1993) 6174.

5. D. J. Burton, R. M. Flynn, J. Fluorine Chem. 10 (1977) 329. 
6. C. F. Bigge, J. T. Drummond and G. Johnson, Tetrahedron Lett. 30 (1989) 7013.

7. (a) G. M. Kosolapoff, J. Am. Chem. Soc. 69 (1947) 1002. (b) G. Kamai, Dokl. Akad. Nauk SSSR 79 (1951) 795; C. A. 46, 6081 (1952). (c) R. Rabinowitz and R. Marcus, J. Am. Chem. Soc. 84 (1962) 1312. (d) R. G. Harvey and E. R. DeSombre, Topics in Phosphorus Chemistry Vol. 1, Wiley, Intersciences New York, (1962) p. 57. (e) A. F. Isbell, U.S. Dept. Com. Office Tech. Serv. Ad 266,695; C. A. 58, 11394 (1963). (f) B. Miller, Topics in Phosphorus Chemistry Vol. 2, Wiley, Intersciences New York, (1965) p. 133. (g) J. Plumb, R. Obrycki and C. Griffin, J. Org. Chem. 31 (1966) 2455. (h) R. Obrycki and C. Griffin, J. Org. Chem. 33 (1968) 632. (i) J. Fu, W. Bentrude and C. Griffin, J. Am. Chem. Soc. 94 (1972) 7717. (j) D. J. Burton and R. M. Flynn, Synthesis (1979) 615.

8. S. Bakkas, M. Juliard and M. Chanon, Tetrahedron 43 (1987) 501.

9. C. U. Kim, B. Y. Luh, P. F. Misco, J. J. Bronson, M. J. M. Hitchcock, I. Ghazzouli and J. C. Martin, J. Med. Chem. 33 (1990) 1207.

10. (a) S. Halazy and V. Gross-Bergès, J. Chem. Soc., Chem. Commun. (1992) 743. (b) D. P. Phillion and D. G. Cleary, J. Org. Chem. 57 (1992) 2763. (c) S. Chen and C. Yuan, Phosphorus, Sulfur, and Silicon 82 (1993) 73. 